\title{
Stability of an Infinite Swept Wing Boundary Layer
}

\section{with Surface Waviness}

\author{
Christian Thomas ${ }^{\mathrm{a}}$, Shahid M. Mughal and Matthew Gipon \\ Department of Mathematics, South Kensington Campus, \\ Imperial College London, London, SW7 2AZ, UK \\ Richard Ashworth and Alejandro Martinez-Cava \\ Airbus Group Innovations, Bristol, BS99 7AR, UK
}

A scheme for generating boundary layers is described and applied to an infinite swept wing model with wavy surface deformations. Steady laminar mean flow is extracted directly from solutions of the Navier-Stokes system of equations, which is validated against computations of a compressible boundary layer method. Furthermore, the routines capture separated boundary layer profiles, overcoming the constraints of the conventional methods. The stability of crossflow disturbances is investigated using both PSE and LNS methods and the effect of chordwise waviness of variable wavelength, amplitude and phase is considered. Wavy surfaces are found to influence the growth of crossflow disturbances, suggesting that the onset of transition may also be affected by the variations in the surface geometry.

a c.thomas@imperial.ac.uk 


\section{Nomenclature}

$$
\begin{aligned}
& A \quad=\text { amplitude of the surface waviness, } \% \text { of } c \\
& C_{p} \quad=\text { pressure coefficient } \\
& C_{f} \quad=\text { skin friction coefficient } \\
& c \quad=\text { chord length normal to the leading edge, } \mathrm{m} \\
& f \quad=\text { frequency of the disturbance, } \mathrm{Hz} \\
& L=\text { wavelength of the surface waviness, } \% \text { of } c \\
& M_{\infty} \quad=\text { freestream Mach number } \\
& N \quad=\mathrm{N} \text { factor } \\
& P \quad=\text { pressure, } \mathrm{Pa} \\
& R_{\infty} \quad=\text { freestream Reynolds number based on the chord } c \\
& R_{C F}=\frac{\left|u_{C F, \max }\right| \delta_{01}}{\nu_{e}}, \text { Crossflow Reynolds number } \\
& T \quad=\text { temperature, } \mathrm{K} \\
& \overline{\mathbf{u}} \quad=\{\bar{u}, \bar{v}, \bar{w}\}, \text { velocity vector in Cartesian } \overline{\mathbf{x}} \text {-coordinates, } \mathrm{m} \cdot \mathrm{s}^{-1} \\
& \mathbf{u}=\{u, v, w\} \text {, velocity vector in wing surface } \mathbf{x} \text {-coordinates, } \mathrm{m} \cdot \mathrm{s}^{-1} \\
& u_{C F, \max }=\text { maximum crossflow velocity, } \mathrm{m} \cdot \mathrm{s}^{-1} \\
& \mathbf{U}=\{U, V, W\} \text {, dimensionless velocity vector in } \mathbf{X} \text {-coordinates } \\
& \mathbf{U}^{\prime} \quad=\left\{U^{\prime}, V^{\prime}, W^{\prime}\right\} \text {, velocity perturbation vector } \\
& \overline{\mathbf{x}}=\{\bar{x}, \bar{y}, \bar{z}\}, \text { Cartesian coordinates, } \mathrm{m} \\
& \mathbf{x}=\{x, y, z\}, \text { wing surface coordinates, } \mathrm{m} \\
& \mathbf{X}=\{X, Y, Z\} \text {, dimensionless chordwise, spanwise, wall-normal coordinates } \\
& \overline{\mathbf{x}}_{n} \quad=\left\{\bar{x}_{n}, \bar{y}_{n}, \bar{z}_{n}\right\} \text {, unit normal vector in Cartesian } \overline{\mathbf{x}} \text {-coordinates } \\
& \rho \quad=\text { density, } \mathrm{kg} \cdot \mathrm{m}^{3} \\
& \nu \quad=\text { viscosity, } \mathrm{Pa} \cdot \mathrm{s} \\
& \delta \quad=\text { boundary layer thickness, } \mathrm{m} \\
& \delta^{*} \quad=\text { displacement thickness, } \mathrm{m} \\
& \delta_{01}=\text { distance from wall where } u_{C F} \text { reduces to } 0.1\left|u_{C F, \max }\right|, \mathrm{m}
\end{aligned}
$$




\section{Nomenclature}

$\theta=$ angle of sweep

$\phi=$ surface transformation angle

$\Lambda=\tan ^{-1}\left(V_{e} / U_{e}\right)$, local angle of the inviscid streamline

$\lambda=$ phase shift of the surface waviness

$\psi=$ orientation of the disturbance

$\beta=$ spanwise wavenumber of the disturbance, $\mathrm{m}^{-1}$

$\alpha=$ dimensionless chordwise growth rate of the disturbance

\section{Subscripts}

$\Delta N=\mathrm{N}$ factor variation due to waviness

$e \quad=$ boundary layer edge

$Z \quad=$ wall-normal first derivative

$Z Z$ = wall-normal second derivative

$\infty=$ freestream flow conditions

\section{Introduction}

An accurate prediction of the extent of laminarity over an aeroplane wing under varying conditions is critical in determining its performance characteristics. In particular, the effect of deformations in the surface under load (waviness, bulges, ridges) or due to environmental conditions (hail stone impacts [1]) can potentially have a signifiant impact on the development of disturbances (Tollmien-Schlichtling (TS) waves, crossflow (CF)) and the location identified as corresponding to laminar flow transition [2]. The structure of the wing with rigid support components (e.g. ribs and stringers) overlaid by a more flexible outer skin, results in wavelike surface deformations along both the chord and spanwise directions with temperature and pressure variations. Differing forms of waviness can characterise the leading edge and the wingbox sections of the wing body. Furthermore, surface waviness can cause localised regions of large adverse pressure gradients that generate flow separation. Thus, it is important that accurate and robust methods are developed that can successfully capture or generate boundary layer profiles for the investigation of disturbance development, stability and transition. 
The effect of various surface deformations on laminar-turbulent transition on a flat plate and on an airfoil were described using wind tunnel experiments by Fage [2]. The height, length and location of the surface bulges or hollows were found to have a significant influence on boundary layer transition. Further experiments on surface waviness included the effects of compressibility, pressure gradients and swept flow [3-5]. Experimental observations were used by Fage to formulate a correlation between the dimensions of the humps/bulges with the flow conditions and the onset of transition. Additionally, Fage defined a minimum height of the surface deformation that would be required to affect the position of boundary layer transition.

Holmes and co-workers [6, 7] reported results of experimental investigations concerning the manufacturing tolerances for natural laminar flow with the effect of steps, gaps and surface wave deformations. The shape of the forward and backward facing steps was found to play an important role in the flow characteristics. Additionally, they reported results of a flight experiment where surface waviness was placed at the leading-edge of the wing. The surface waviness generated a large adverse pressure gradient that destabilised the TS wave instability. Wang and Gaster [8] conducted an experimental study on a flat plate to investigate the effect of a sharp-edged step on TS waves and transition. Increasing the step height was found to amplify the growth of the TS instability, causing the premature onset of transition

Qualitatively similar stability and transition characteristics were observed in theoretical investigations concerning singular bumps on flat plates [9-12]. Linear stability theory was carried out using a parallel flow based approximation of the boundary layer, where the streamwise variation of the meanflow was ignored. Boundary layer transition was then predicted using a locally based $e^{N}$ procedure [13-16]. Wie \& Malik [17] conducted a stability analysis of surface waviness effects on TS instabilities and transition in a two-dimensional subsonic flow over a flat plate. Their analysis was based on a non-parallel boundary layer that took account of the streamwise variations of the meanflow, while linear stability was computed using parabolized stability equation (PSE) methods [18]. Various wave configurations and compressible flow conditions were considered and it was determined that the height and length of the surface wave were critical to the stability and transition calculations, providing agreement with the earlier experimental observations. 
PSE methods were further utilized in the analysis of humps on a two-dimensional flat plate $[19,20]$. The height and length scales of the hump were again critical to the amplification rates of the established disturbances, with significant increases in the $N$-factor observed when the hump width was reduced. An investigation of nonlinear effects on TS wave instabilities and transition breakdown, demonstrated that in the presence of a hump, nonlinear interaction could cause significant amplification of the disturbance, even for very small initial amplitudes. This would suggest that a hump of sufficient size could cause the premature breakdown of the laminar flow.

Industrial methods for transition prediction on conventional fixed wing aircraft typically make an assumption of conical flow based on a locally swept tapered approximation of the geometry. This enables a strip based analysis where the base flow and the stability equations are solved on a twodimensional plane across the wing-span. Mughal [21] computes the meanflow over a swept wing by solving the system of compressible boundary layer equations (from here on denoted as CoBL) using a streamwise marching procedure [22], where the surface pressure coefficient $C_{p}$ is typically required as an initial flow condition. (Further details regarding the input are given in the subsequent section). A fully implicit second-order accurate three-point backward differencing method was implemented along the streamwise direction, whilst a two point second-order accurate scheme was utilised in the wall normal direction. Base flow solutions of the CoBL set of equations have previously been utilised for both PSE and receptivity analysis [23, 24], for a range of model flow systems. This particular method for generating boundary layer flow profiles is also utlised in this investigation as a means of validating, where possible, a new approach for generating boundary layers.

Boundary layer solvers are very useful for computing flow profiles and enabling the analysis of boundary layer instabilities. However, they do neglect some flow characteristics, including the effects of surface curvature. Furthermore, as solutions of the boundary layer equations are based on streamwise marching procedures [22], difficulties will inevitably be encountered when the flow separates from the body surface, as this causes the break down of the numerical method. Thus, stability investigations that utilise boundary layer solvers are restricted to the flow range upstream of the separated region. For many flow systems this is not necessarily a problem. However, for flows that experience dramatic variations in pressure (that may be caused by the surface waviness that 
is investigated in this paper) regions of separation can form. Thus, new methods for computing boundary layer profiles are required.

Interactive boundary layer (IBL) methods [25] have been developed that can compute the flow solutions of separated boundary layers. The procedure generates flow profiles by accounting for the viscous-inviscid flow interaction, where the outer flow and boundary layer equations are solved simultaneously. IBL methods were implemented by Wie \& Malik [17] in their investigation of the effect of surface waviness on two-dimensional TS wave instabilities. However, in that particular investigation their stability analysis was restricted to only attached flow systems. Although IBL methods have been successfully utilised to investigate flows with boundary layer separation, they are not without their own shortcomings. For instance, the inviscid-viscous interaction law can make flow assumptions based on thin airfoil theory [17].

For the current investigation an alternative scheme for generating boundary layer profiles is described and applied to a swept wing body with variable wavy surface deformations. The method is based on flow solutions that are established by an industrial direct numerical simulation solver called TAU [26]. The TAU program solves the RANS system of equations and computes steady three-dimensional flow solutions about a swept wing that is embedded within an unstructured mesh. However, steady laminar boundary layers can be obtained by specifying transition about a fixed chord position. Thus, the RANS solver reduces to the Navier-Stokes system of equations, which compute laminar velocity, pressure and temperature fields across the span of the wing model. If the TAU mesh is chosen to be sufficiently dense within the region of the boundary layer, the resulting TAU solutions can capture comprehensive flow characteristics that may include regions of separation. Boundary layers can then be constructed by carefully extracting flow properties directly from the three-dimensional computations. This is achieved using "RANS extracted boundary layers" (REBL) that transforms and scales the TAU computations. As solutions are drawn directly from the TAU output, boundary layers that include regions of separation can then be investigated. Thus, the REBL extraction scheme avoids the drawbacks of conventional boundary layer methods that breakdown due to the appearance of separation.

A similar approach for generating boundary layers from Navier-Stokes solutions has been 
utilised by Malik [27] and Liao et al [28] amongst others to analyse the stability of complex flows. For instance, the latter investigators developed a procedure for extracting mean flows from an unstructured-grid on both a flat plate and a full aircraft configuration. Nevertheless, although our REBL extraction scheme is not new per se, it is the first attempt at directly coupling solutions of the TAU flow solver with the PSE and LNS methods developed by Mughal [23, 24].

In the subsequent section the REBL procedure is introduced and used to investigate the stability of $\mathrm{CF}$ disturbances on a swept wing body. A secondary boundary layer scheme, CoBL, is utilised when possible, as a reference solution for validating the REBL computations. The results generated by the two boundary layer methods are then used as inputs for a stability analysis of CF instabilities that is based on non-parallel PSE and linearised Navier-Stokes (LNS) methods. The effect of wavy surface variations, on the development and growth of $\mathrm{CF}$ instabilities in a compressible flow, are then investigated by imposing a sinusoidal waviness along the chord direction of variable wavelength, amplitude and phase. Furthermore, the stability analysis is extended to those flow systems where the wavy surface has engineered boundary layer separation. For brevity the reader is referred to Mughal and co-workers $[23,24]$ for a complete and detailed account of the PSE and LNS schemes. Thus, only the necessary ingredients for stability analysis are discussed herein.

\section{RANS Extracted Boundary-Layer (REBL)}

\section{A. Swept wing model and Compressible Boundary Layer Method}

Figure 1 illustrates the M2355 infinite swept wing model [29] that is used in this investigation to examine the effects of surface waviness on the growth of CF instabilities. The model was implemented within the flow solver TAU with a mesh that was chosen to be sufficiently dense to fully capture the wall-normal variations of the boundary layer. This was achieved by using at least 40 mesh points within the boundary layer. A swept wing model was then established for an angle of sweep $\theta=25^{\circ}$ with the freestream conditions given as $\left\{R_{\infty}, u_{\infty}, M_{\infty}\right\}=\left\{5 \times 10^{6}, 243,0.7\right\}$, while

a laminar flow was obtained along the leading half of the wing model by specifying a transition line about $0.6 c$. Downstream of this location the flow was treated as being turbulent and was ignored for this investigation.

The TAU solver simulates the flow that develops adjacent to the M2355 model in a Cartesian 


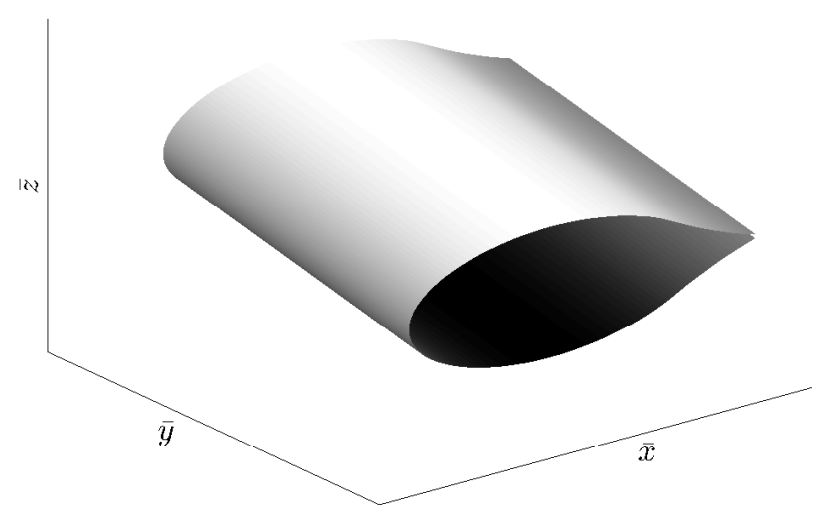

Fig. 1 Illustration of the M2355 infinite swept wing model [29].

coordinate system $\overline{\mathbf{x}}=\{\bar{x}, \bar{y}, \bar{z}\}$. A converged steady state solution was then obtained by solving the RANS formula that are given by the Spalart-Allmaras-Edwards turbulence model [30]. For the laminar region, the eddy viscosity was set to zero. Thus, the RANS formulation reduces to the Navier-Stokes equations that are solved subject to the no-slip conditions on the wing surface and farfield boundary conditions given by the above freestream specifications. The TAU solver then implements an explicit Runge-Kutta iterative scheme, where we assume that the steady state solution is fully converged once residuals are of order $10^{-8}$.

Figure 2 depicts a cross-sectional $(\{\bar{x}, \bar{z}\}$-plane) and aerial view $(\{\bar{x}, \bar{y}\}$-plane $)$ of the M2355 swept wing body inclined at an angle $\theta$. For simplicity it was assumed that the flow is independent of the spanwise direction. Under this assumption, for a surface coordinate system $\mathbf{x}=\{x, y, z\}$, the basic equations for the development of a compressible boundary layer are given as

$$
\begin{gathered}
\frac{\partial(\rho u)}{\partial x}+\frac{\partial(\rho w)}{\partial z}=0 \\
\rho\left(u \frac{\partial u}{\partial x}+w \frac{\partial u}{\partial z}\right)=\rho_{e} u_{e} \frac{d u_{e}}{d x}+\frac{\partial}{\partial z}\left(\mu \frac{\partial u}{\partial z}\right) \\
\rho\left(u \frac{\partial v}{\partial x}+w \frac{\partial v}{\partial z}\right)=\frac{\partial}{\partial z}\left(\mu \frac{\partial v}{\partial z}\right) \\
\rho\left(u \frac{\partial T}{\partial x}+w \frac{\partial T}{\partial z}\right)=\frac{1}{\sigma} \frac{\partial}{\partial z}\left(\mu \frac{\partial T}{\partial z}\right)-\left(\frac{\rho_{e} u_{e}}{C_{p}} \frac{d u_{e}}{d x}\right) u+\frac{\mu}{C_{p}}\left\{\left(\frac{\partial u}{\partial z}\right)^{2}+\left(\frac{\partial v}{\partial z}\right)^{2}\right\} .
\end{gathered}
$$



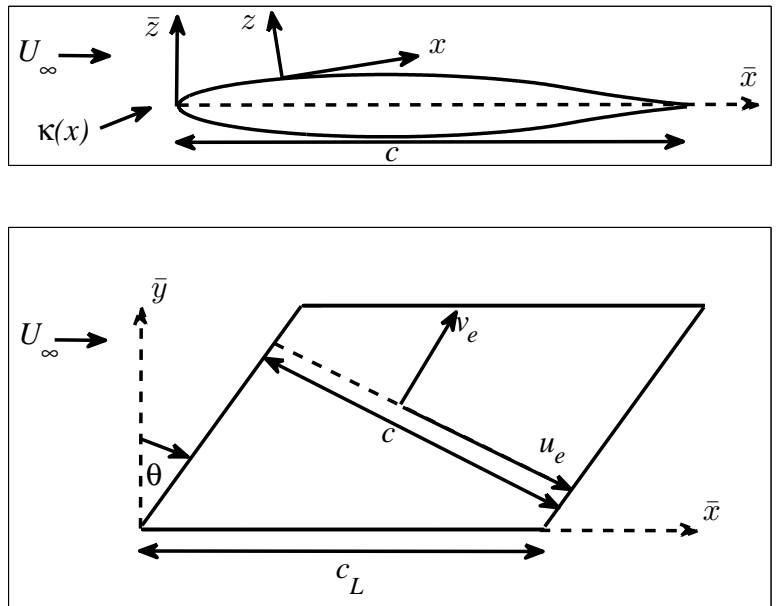

Fig. 2 Diagram illustrating the cross-sectional and aerial view of the infinite swept wing model.

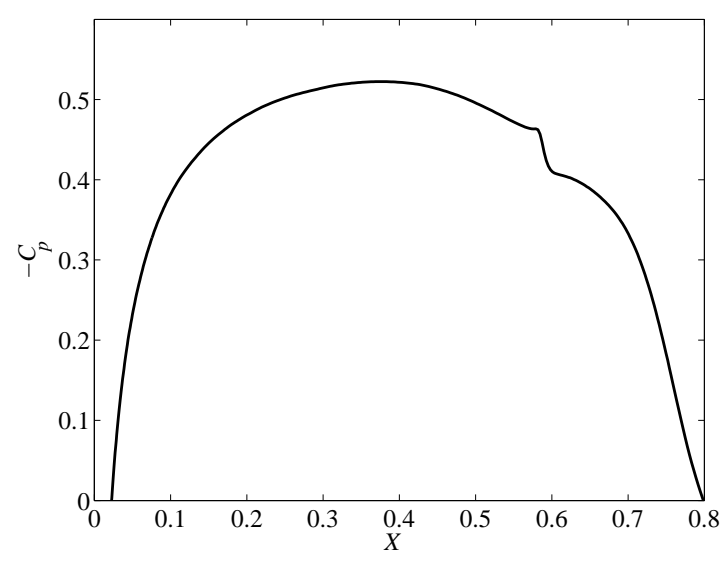

Fig. 3 Surface pressure coefficient $C_{p}$ against the chord direction $X$ for a $25^{\circ}$ swept wing model. Initial flow conditions given as: $R_{\infty}=5 \times 10^{6}, u_{\infty}=243$ and $M_{\infty}=0.7$.

This system of compressible boundary layer equations are solved using the streamwise marching method CoBL $[21,22]$, where the pressure coefficient $C_{p}$ is required as a parameter input. The variation of $C_{p}$ along the surface of the non-deformed M2355 swept wing is depicted in figure 3 as a function of the non-dimensional chord $X(\equiv x / c)$-direction. The magnitude of $C_{p}$ grows as it passes over the wing model attaining an absolute maximum near $X=0.4$ before dropping sharply about the specified transition line that is located about $X=0.6$. 
(a)

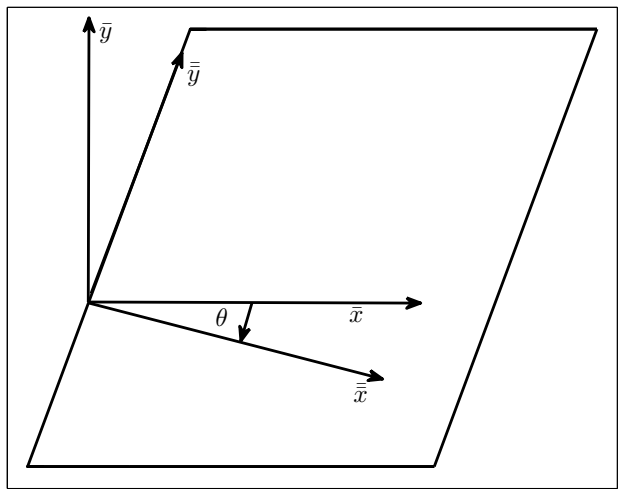

(b)

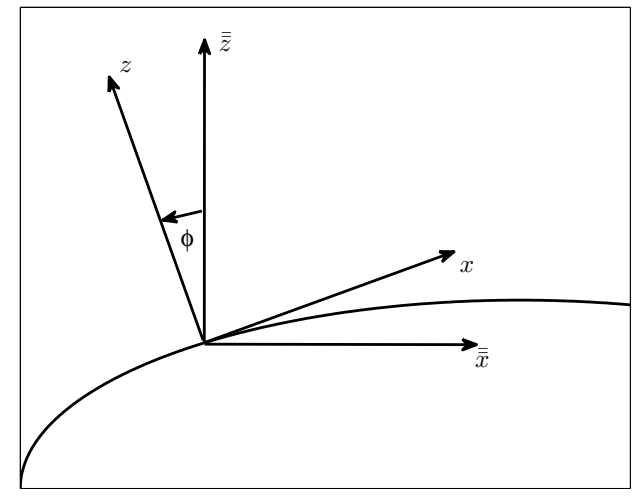

Fig. 4 Flow transformations required to transform flow properties into the body fitted coordinate system. (a): Angle of sweep $\theta$. (b): Angle $\phi$ made with surface.

(a)

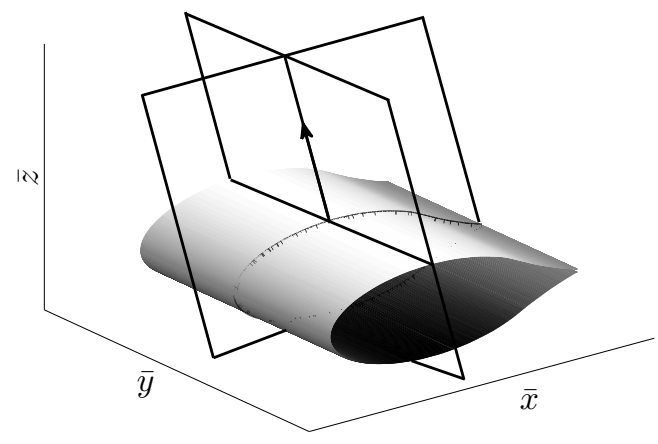

(b)

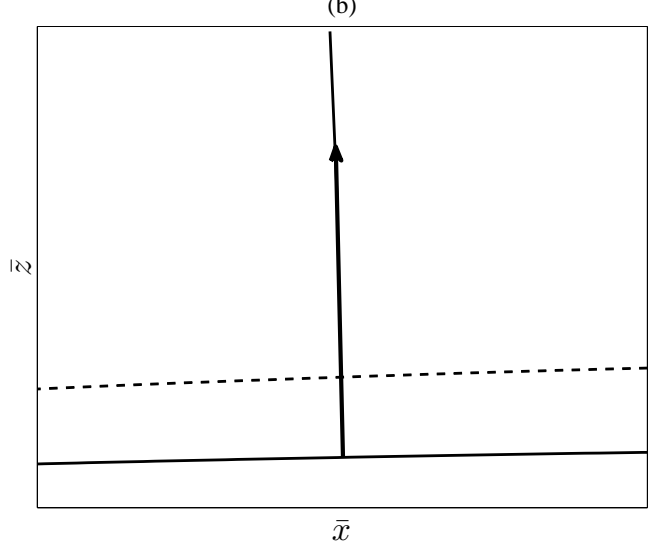

Fig. 5 Illustrations of the boundary layer extraction from the RANS solutions. (a): Solutions extracted along surface normals (arrow) that is given as the line intersection of two planes. (b): Boundary layer edge characteristics estimated using the total pressure of the flow system (or Bernoulli's Theorem), represented as a dashed contour.

\section{B. Coordinate Transformation and Estimating the Boundary-Layer Edge}

The TAU flow solutions are originally computed within Cartesian coordinates, but are transformed by REBL into a surface coordinate system (as illustrated in the cross-sectional view in figure 2). This is achieved by implementing simple geometric transformations and boundary layer properties. Paraview filters [31] are utilised that perform several operations during the boundary layer extraction process, while arithmetic operations are performed using the programming language 
Python to the default 15 decimal place accuracy.

Unit normals are generated at all points on the surface, along a line normal to the leadingedge of the wing. Transformation angles are then required to convert the flow solutions from the Cartesian to the surface coordinates. These are defined on the angle of sweep $\theta$ (as depicted in figure 4(a)) and secondary transformations based on the unit normal $\overline{\mathbf{x}}_{n}$. Figure $4(\mathrm{~b})$ illustrates the latter transformation, $\phi=\sin ^{-1}\left(\bar{x}_{n}\right)$, while a third angle of transformation can be defined in terms of the normal along the $\bar{y}$-direction. However, we assume that the three-dimensional undisturbed flow $\mathbf{U}=\{U, V, W\}$ is only a function of the $x$ - and $z$-directions and is independent of the spanwise $y$ direction. Thus, this latter transformation angle is neglected as it is assumed that $\bar{y}_{n}=0$. Boundary layer solutions are then obtained by extracting data directly along the line given by the unit normal $\overline{\mathbf{x}}_{n}$ (as indicated by an arrow in figure $5(\mathrm{a})$ ), which is given as the intersection of two planes (also illustrated in figure 5(a)) that are constructed at each surface point using plane normals based on the surface transformation angle $\phi$ :

$$
\{\cos \phi, 0, \sin \phi\} \quad \text { and } \quad\{0,1,0\}
$$

Steady laminar TAU solutions are also given in dimensional quantities, but for stability investigations, flow profiles are required in a non-dimensional format. Thus, scale factors are necessary to non-dimensionalise velocity, temperature and length fields. Suitable scalings are defined on the flow characteristics at the boundary layer edge. However, the TAU flow solver does not directly measure the properties at the edge of the boundary layer. Nevertheless, appropriate flow scales are obtained by defining the boundary layer thickness as $99 \%$ of the total pressure (dashed curve in figure $5(\mathrm{~b})$ ). This particular flow field represents the sum of the static and dynamic pressures and corresponds to Bernoulli's principle. Flow properties at the estimated boundary layer edge are then extracted to formulate normalisation factors; the chordwise velocity $u_{e}$, the pressure $P_{e}$, the temperature $T_{e}$ and a locally defined length scale $\sqrt{\frac{c x \nu_{e}}{u_{e}}}$. Solutions are then transformed and scaled to give the non-dimensional velocity and temperature profiles

$$
\mathbf{U}=\frac{\mathbf{u}}{u_{e}} \text { and } \frac{T}{T_{e}}
$$



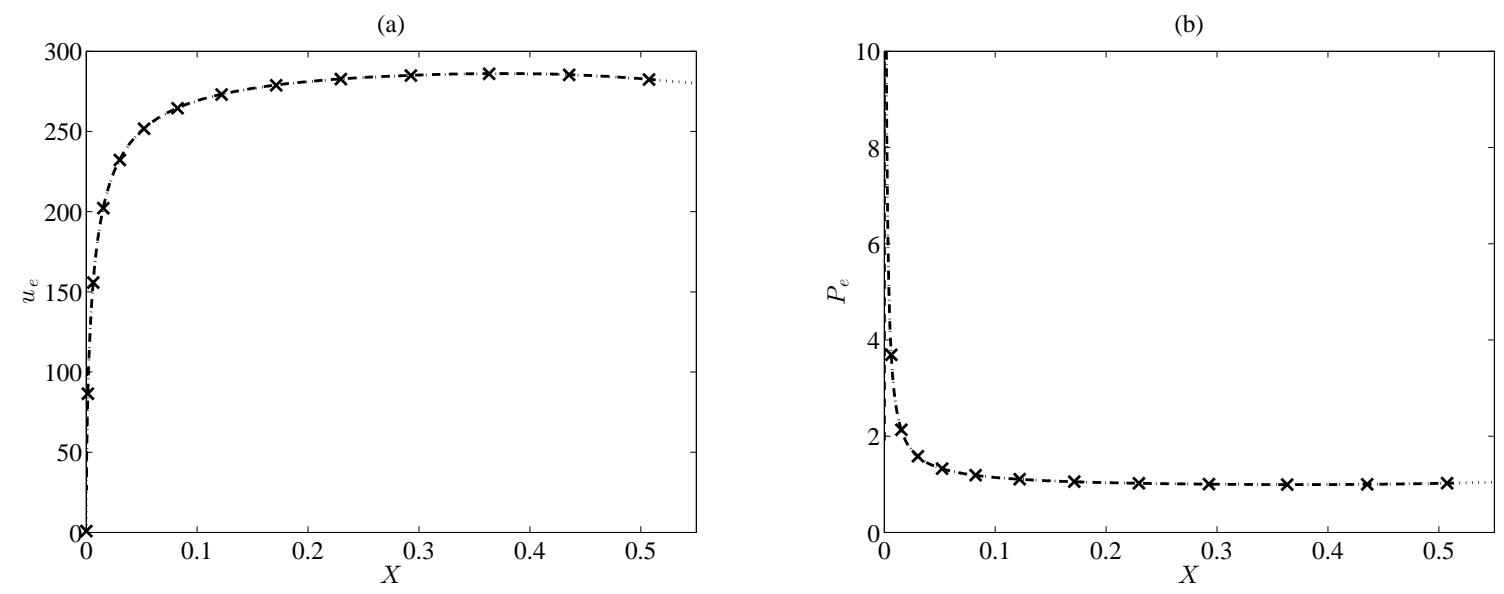

Fig. 6 Flow characteristics for an infinite swept wing with a $25^{\circ}$ angle of sweep. Dashed lines represent the input for the CoBL scheme, while dotted lines with cross markers depict results given by REBL. (a): Boundary layer edge velocity $u_{e} \cdot(\mathbf{b})$ : Boundary layer edge pressure $P_{e}$. where $\mathbf{u}=\overline{\mathbf{u}}\{\theta, \phi\}$, for

$$
\left[\begin{array}{l}
u \\
v \\
w
\end{array}\right]=\left[\begin{array}{ccc}
\cos \theta \cos \phi & -\sin \theta \cos \phi & \sin \phi \\
\sin \theta & \cos \theta & 0 \\
-\cos \theta \sin \phi & \sin \theta \sin \phi & \cos \phi
\end{array}\right]\left[\begin{array}{c}
\bar{u} \\
\bar{v} \\
\bar{w}
\end{array}\right] .
$$

Similar transformations are used to formulate expressions for the velocity and pressure gradients. For instance the non-dimensional pressure gradient $P_{X}$ along the chordwise $X$-direction is defined as

$$
P_{X}=\left(\cos \theta \cos \phi \bar{P}_{\bar{x}}-\sin \theta \cos \phi \bar{P}_{\bar{y}}+\sin \phi \bar{P}_{\bar{z}}\right) /\left(\rho_{e} u_{e}^{2}\right),
$$

for $\nabla \bar{P}=\left\{\bar{P}_{\bar{x}}, \bar{P}_{\bar{y}}, \bar{P}_{\bar{z}}\right\}$ the pressure gradient in dimensional Cartesian coordinates. Finally, the non-dimensional boundary layer profiles are cubic spline fitted along the wall-normal $z$-direction on to a linearly distributed mesh $Z=z / \sqrt{\frac{c x \nu_{e}}{u_{e}}} \in[0: 16]$.

\section{Analysis of Non-Deformed Model}

Flow characteristics at the edge of the boundary layer are drawn in figure 6 , with dashed lines representing the input for the CoBL scheme and dotted lines depicting the corresponding REBL solutions. Cross markers have also been used to represent the results given by REBL to help delineate between the two sets of calculations. The boundary layer edge velocity $u_{e}$ (figure 6(a)) 

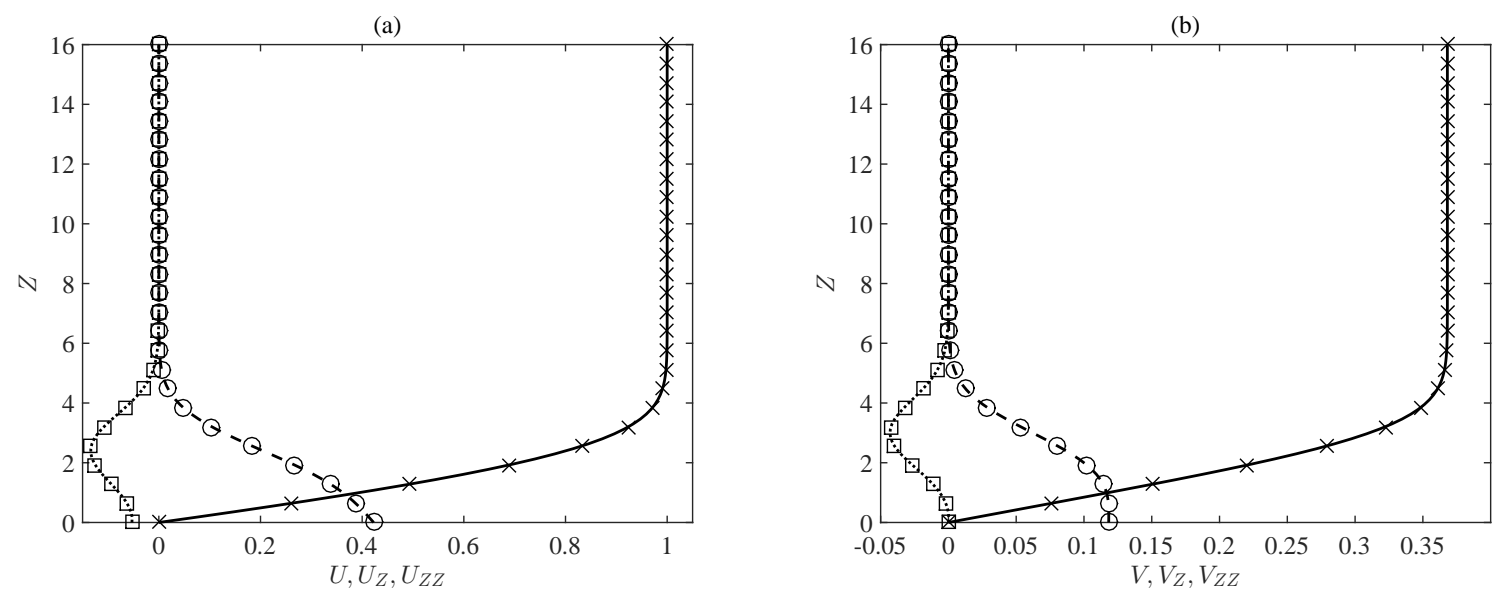

Fig. 7 Boundary layer velocity comparisons at $X=0.2$ for an infinite swept wing with a $25^{\circ}$ angle of sweep. (a): Non-dimensional chord velocity $U$ (solid-CoBL, cross markers-REBL), $U_{Z}$ (dashed-CoBL, circle markers-REBL) and $U_{Z Z}$ (dotted-CoBL, square markers-REBL). (b): Non-dimensional spanwise velocity $V$ (solid-CoBL, cross markers-REBL), $V_{Z}$ (dashed-CoBL, circle markers-REBL) and $V_{Z Z}$ (dotted-CoBL, square markers-REBL).

Table 1 Comparisons between the CoBL and REBL boundary layer schemes at several chord positions.

\begin{tabular}{cccc}
\hline \hline$X$ & $U_{Z}(0)$ & $V_{Z}(0)$ & $V(Z \rightarrow \infty)$ \\
\hline $0.05(\mathrm{CoBL})$ & 0.6263 & 0.1645 & 0.4322 \\
$0.05(\mathrm{REBL})$ & 0.6323 & 0.1670 & 0.4343 \\
$0.1(\mathrm{CoBL})$ & 0.5045 & 0.1341 & 0.3893 \\
$0.1(\mathrm{REBL})$ & 0.5054 & 0.1346 & 0.3895 \\
$0.2(\mathrm{CoBL})$ & 0.4226 & 0.1182 & 0.3681 \\
$0.2(\mathrm{REBL})$ & 0.4230 & 0.1184 & 0.3682 \\
$0.3(\mathrm{CoBL})$ & 0.3792 & 0.1121 & 0.3615 \\
$0.3(\mathrm{REBL})$ & 0.3792 & 0.1121 & 0.3616 \\
\hline \hline
\end{tabular}

and pressure $P_{e}$ scalings (figure $\left.6(\mathrm{~b})\right)$ are plotted against the scaled chord $X$-direction. Remarkably, there are no discernible differences between the CoBL and REBL solutions, which illustrates the accuracy of the REBL method in estimating the edge of the boundary layer.

Velocity profiles about $X=0.2$ are depicted in figure 7 against the non-dimensional wall-normal 
(a)

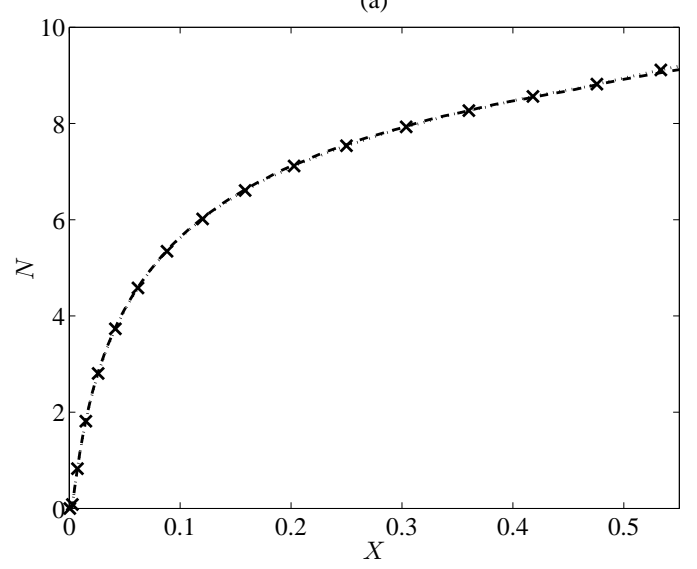

(c)

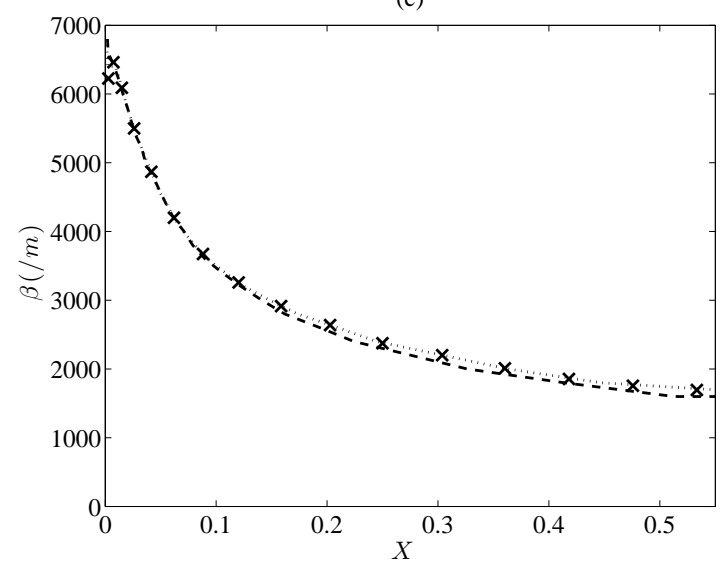

(b)

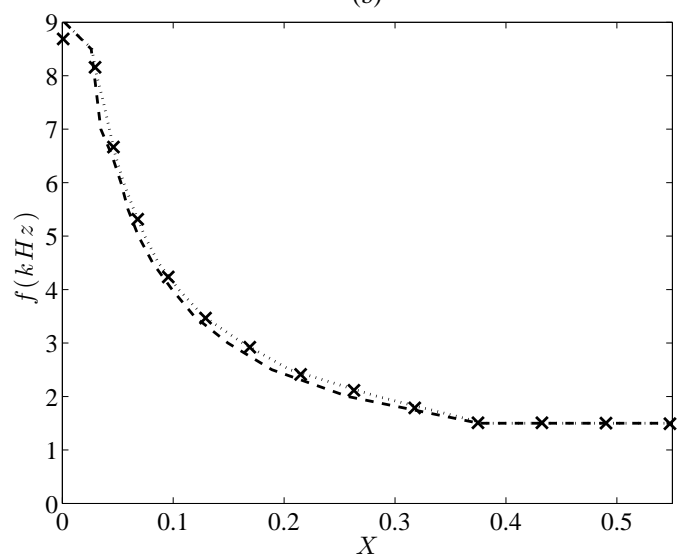

(d)

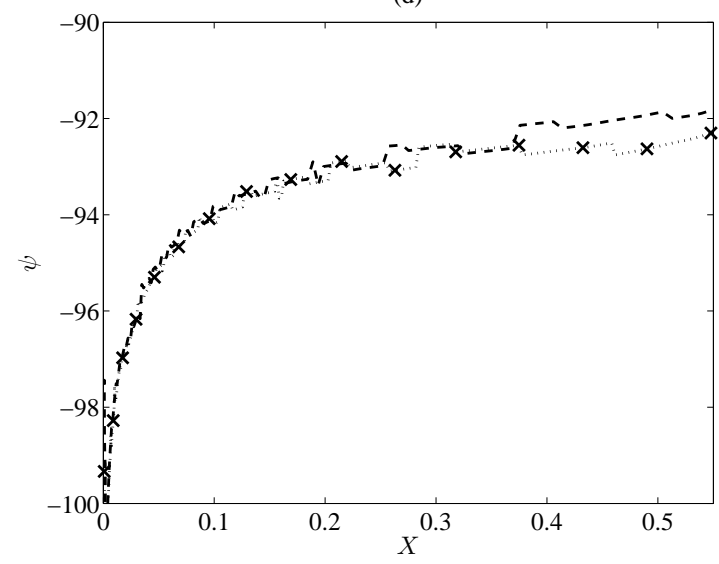

Fig. 8 PSE analysis comparing CoBL (dashed lines) and REBL (dotted with cross markers) solutions. (a): $N$-factor; (b): Frequency $f ;(\mathbf{c})$ : Spanwise wavenumber $\beta$; (d): Angle of orientation $\psi$.

$Z$-axis. Both chordwise $U$ - and spanwise $V$-velocities are drawn in the figure with their associated non-dimensional wall-normal first and second $Z$-derivative components. The two sets of boundary layer solutions are in such good agreement that it is again almost impossible to distinguish between the line/marker types of matching velocity variables. In order to facilitate the excellent agreement between the solutions of CoBL and REBL, table 1 compares several flow properties at four locations along the chord $X$-direction. The small variations in computations near the leading-edge (small $X$ ) can be attributed to the CoBL method ignoring curvature effects that are included in the solutions of the TAU solver and extracted by the REBL procedure.

Non-parallel PSE analysis [23] was then applied to the boundary layer solutions generated by the CoBL and REBL schemes, with equally excellent agreement achieved for the CF stability calcu- 
lations. The effects of surface curvature on the growth of disturbances have been neglected for simplicity, while linear stability analysis was implemented by assuming that the velocity perturbations $\mathbf{U}^{\prime} \ll \mathbf{U}$. Figure 8(a) displays and compares the $N$-factor computations based on $e^{N}$ measurements, which have been constructed by comparing disturbances of many frequencies $f \in[1000: 10000]$ and spanwise wavenumbers $\beta \in[1000: 10000]$. The strongest growing $\mathrm{CF}$ mode at each position $X$ is then used to draw the illustrated calculations, while the analysis of small spanwise wavenumbers $\beta$ has been deliberately neglected to ensure that the investigation concerns only the growth of $\mathrm{CF}$ disturbances and not the two-dimensional TS wave instabilities that may develop in regions with a strong adverse pressure gradient. The associated strongest growing frequency $f$ (measured in $k H z)$, spanwise wavenumber $\beta\left(m^{-1}\right)$ and orientation angle $\psi$ of the disturbance are also included in figure 8. Dashed lines depict PSE analysis based on the CoBL flow solution and the dotted lines with cross markers illustrate the corresponding computations given for the REBL generated boundary layer. Differences between the CoBL and REBL results are again marginal and further improvements could be achieved by increasing the number of mesh points within the boundary layer of the TAU solution.

\section{Surface Deformations}

\section{A. Effects on Boundary Layer Characteristics}

Surface deformations are modelled on the M2355 infinite swept wing as sinusoidal waviness along the chord $X$-direction

$$
A \cos \{2 \pi X / L+\lambda\}
$$

where the wavelength $L$ and amplitude $A$ are given as a percentage of the chord length $c$. The waviness is also characterised by a phase shift $\lambda \in[-\pi: \pi]$ that starts at the leading-edge of the wing model. Figure 9 depicts an exaggerated illustration of the form of the waviness that is established along the surface of the model. For more physically relevant deformations, figure 10 displays the effect of surface waviness on the development of $C_{p}, u_{e}, \delta^{*}$ and $C_{f} R_{\infty}^{1 / 2}$ that are obtained by applying REBL to flow solutions generated by TAU. The freestream flow conditions are unchanged from the above analysis and for simplicity are fixed for the remainder of this investigation. Four surface 


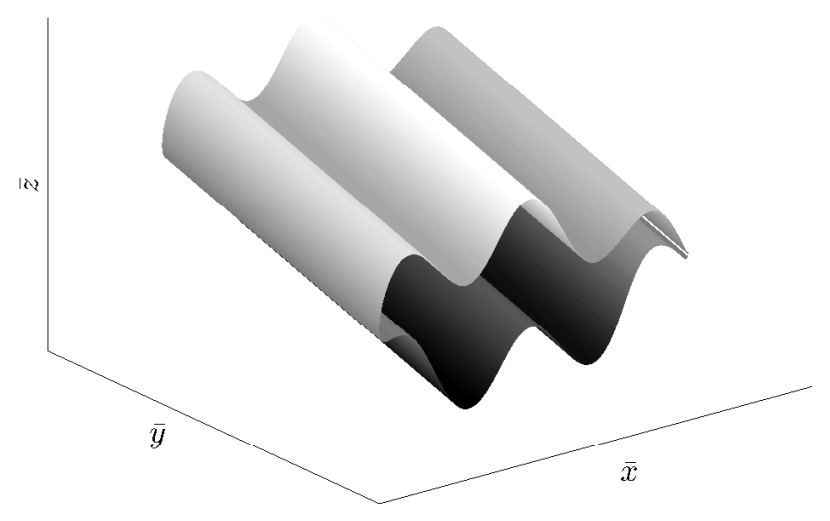

Fig. 9 Illustration of an exaggerated chordwise waviness on the M2355 infinite swept wing model.

configurations are considered with the phase shift $\lambda=0$, where the solid line represents the case $A=L=0$, dashed $\{A, L\}=\{0.01,10\}$, chain $\{A, L\}=\{0.02,20\}$ and dotted $\{A, L\}=\{0.04,40\}$. For a chord $c$ of dimensional length of one metre, these particular specifications would establish sinusoidal wavy walls with amplitudes of size 0.1 through to $0.4 \mathrm{~mm}$. The results demonstrate that variable surface configurations can have a significant effect on the evolution of the flow and it would appear that the sinusoidal waviness of the wing wall has been mirrored in the flow properties. The illustrated flow parameters display comparable wavy characteristics, with relatively large variations from the results obtained for the non-deformed swept wing (solid lines). The surface waviness causes the skin friction coefficient $C_{f} R_{\infty}^{1 / 2}$ to oscillate, increasing and decreasing over localised chord intervals, which (as we might have predicted) coincide with the flow passing over the peaks and troughs of the wavy surface. If we then consider surface configurations with a larger amplitude $A$ (as carried out in the subsequent analysis), the skin friction coefficient will eventually become negative over some sections of the wing. Thus, the associated adverse pressure gradient will have become sufficiently large to cause negative skin friction and boundary layer separation. 
(a)

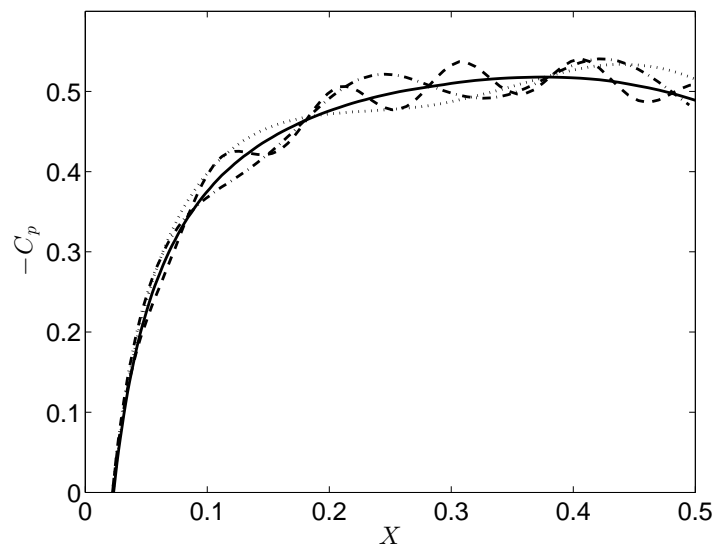

(c)

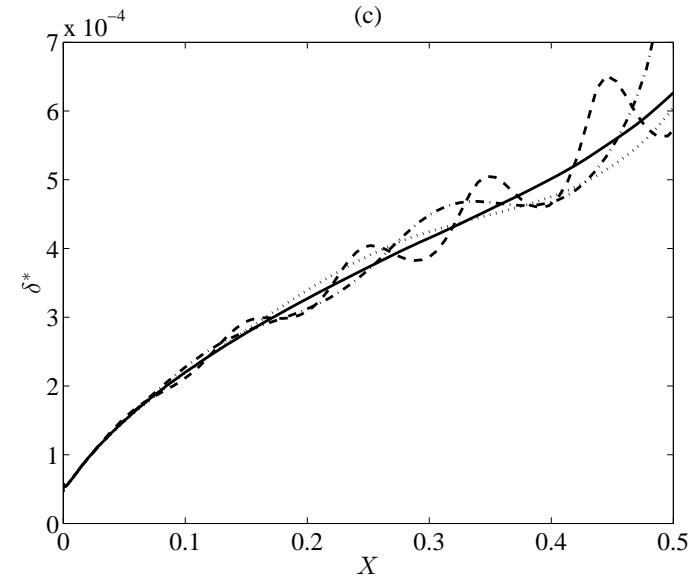

(b)

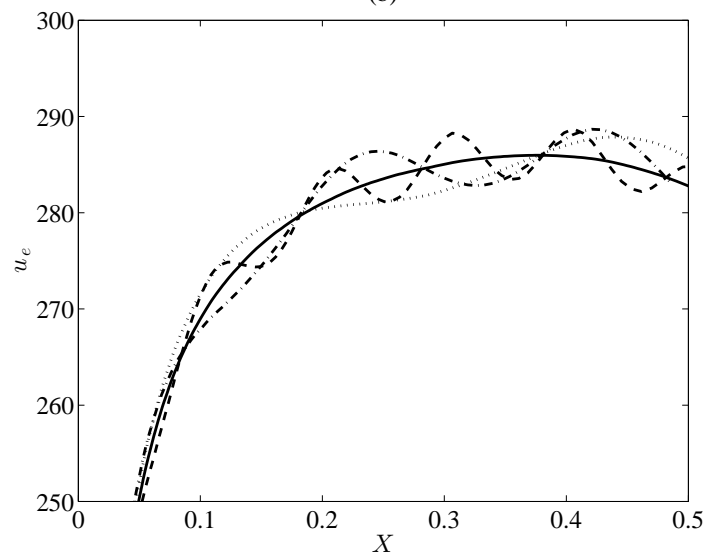

(d)

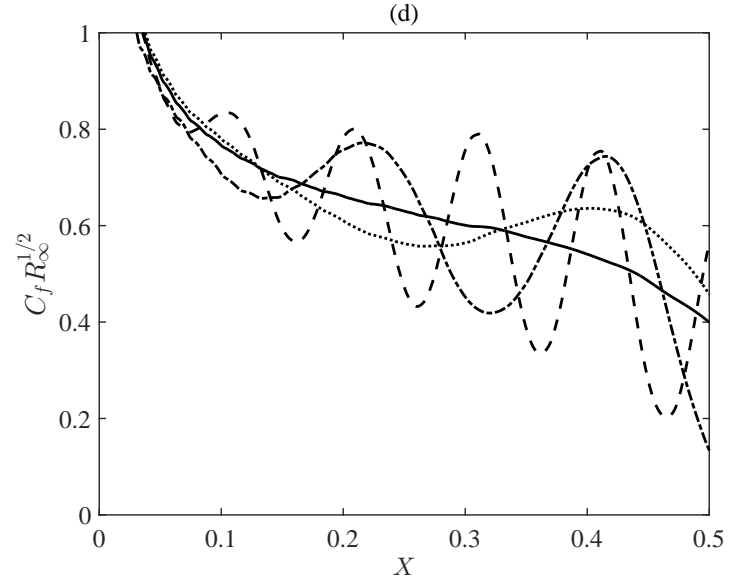

Fig. 10 Effects of chordwise surface waviness on (a): Pressure coefficient $C_{p}$. (b): Boundary layer edge velocity $u_{e} .(\mathrm{c})$ : Displacement thickness $\delta^{*}$. (d): Skin friction $C_{f} R_{\infty}^{1 / 2}$. Solid line represents the non-deformed model, while dashed, chain and dotted lines represent the respective waviness specifications $\{A, L\}=\{0.01,10\},\{0.02,20\},\{0.04,40\}$.

\section{B. Separated Flows}

Figure 11 illustrates solutions of the non-dimensional chordwise $U$-velocity field within the $\{X, z\}$-plane that have been obtained using the REBL extraction scheme. (Note that the flow solutions are drawn using the dimensional vertical $z$-axis to help visualise the physical sizes and variations of the wavy surface). The wavelength of the sinusoidal waviness $L=10$ and the amplitude $A$ increases in successive subplots of figure 11 as $0.0,0.01,0.02$ and 0.04 . Velocities $U$ are depicted from values of zero or marginally negative (blue) near the wall to a maximum value in the far field region (red). The dimensional $z$-axis has been deformed and scaled to help visualise the relative sizes of the wavy surface variations and the resulting impact on the flow development. It is worth 

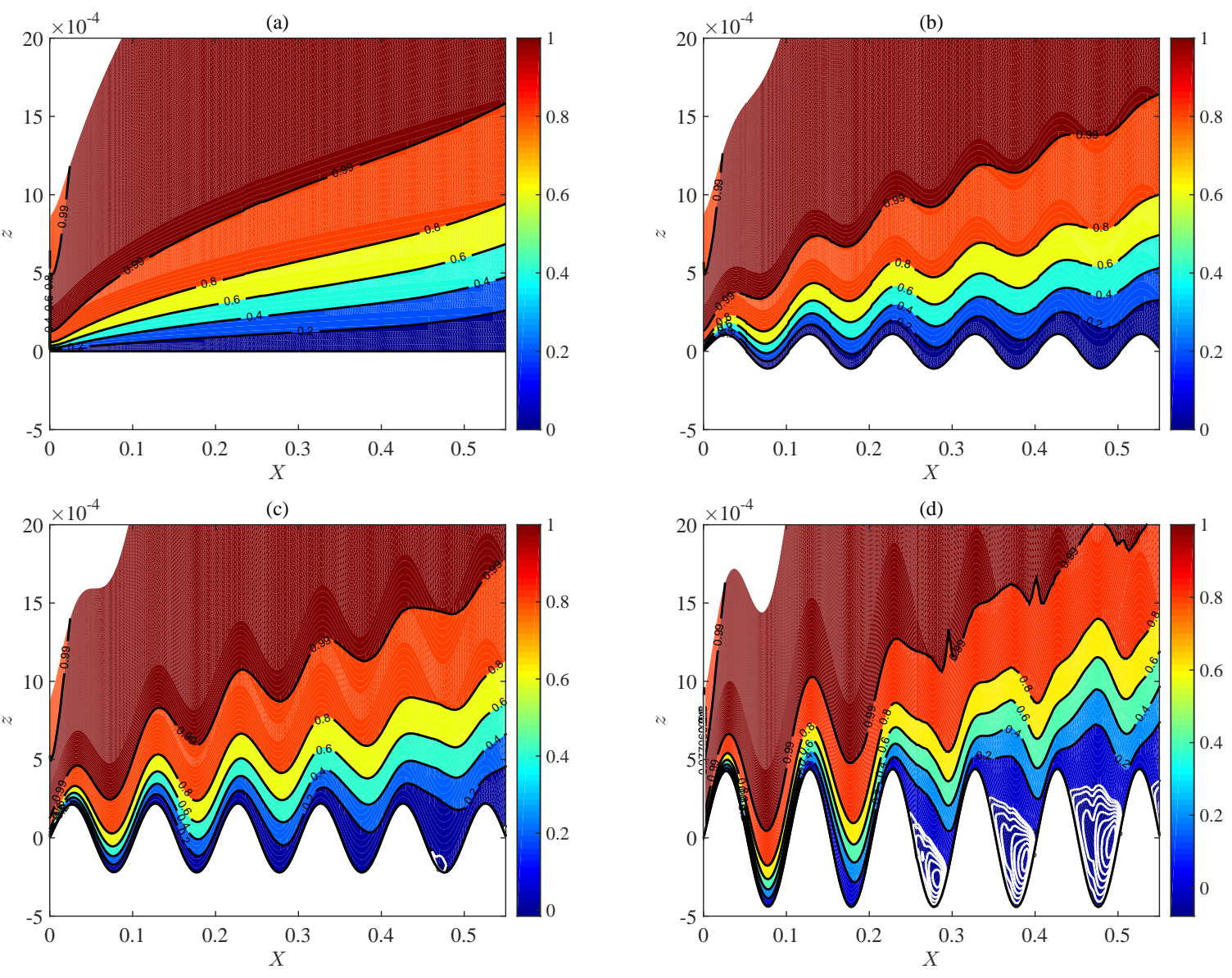

Fig. 11 Contour representation of the non-dimensional $U$-velocity field in the $\{X, z\}$-plane for $L=10$ and $A=0,0.01,0.02,0.04$. Blue colours represent flow values on or near the surface of value 0 (in a, b) and -0.02 (c) and -0.08 (d), while dark red colours specify the maximum size of the velocity profile beyond the boundary layer edge. Solid white contour lines in (c, d) indicate regions of flow separation.

noting that the vertical axis is measured in millimetres, whilst the horizontal axis represents length scales per unit metre. Solid white contour lines in plots 11(c, d) highlight regions of reverse flow that are assumed to be steady and increasingly complex as the amplitude $A$ increases. It should be noted that in regard to the latter two illustrations, the CoBL scheme for generating boundary layers would be unable to draw equivalent complete images, as the method would breakdown before the onset of the first separation bubble.

Increasing the amplitude of the waviness results in substantial variations along the wall-normal direction in both the velocity field and the boundary layer thickness $\delta$. As the flow develops down- 

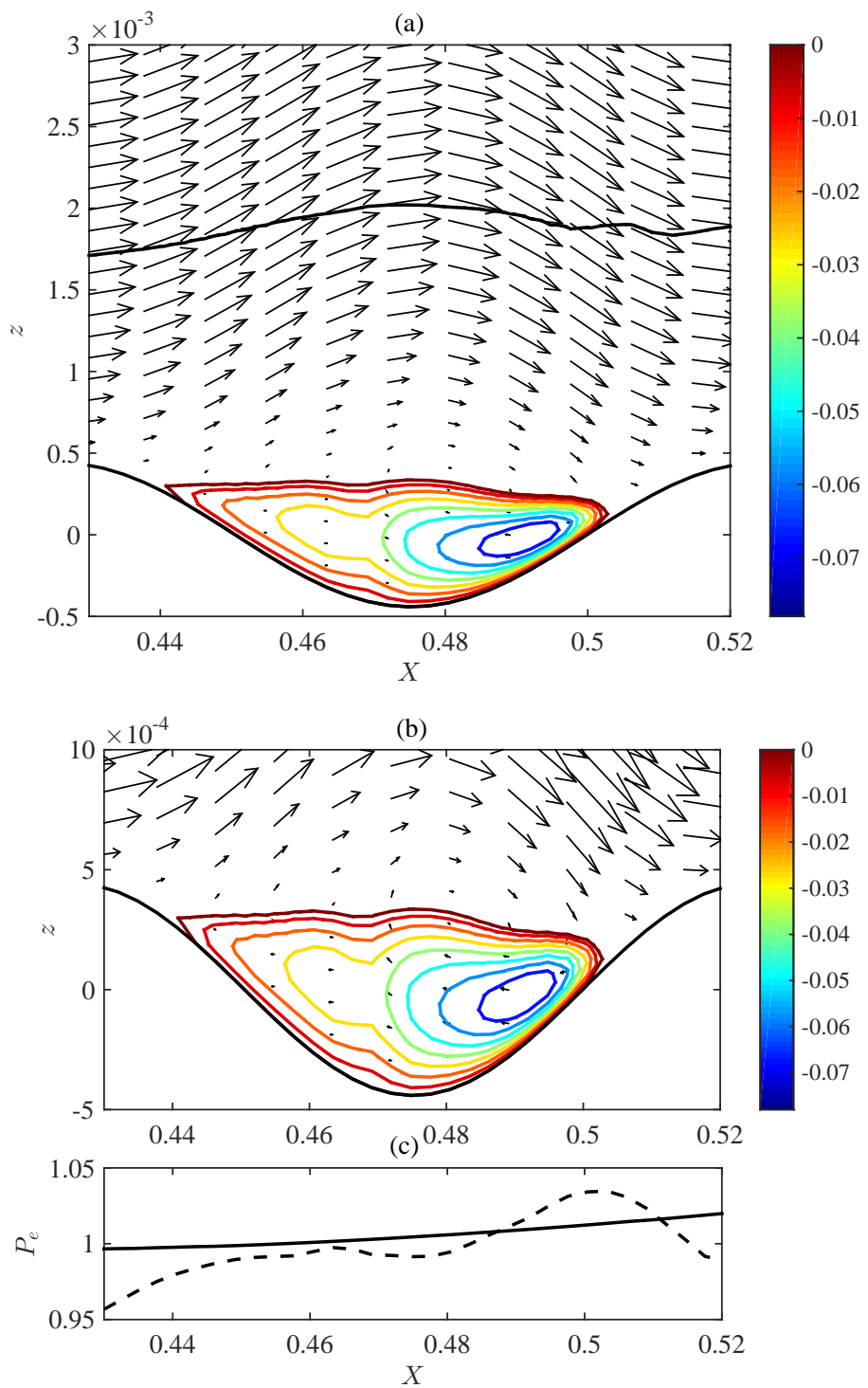

Fig. $12(\mathrm{a}, \mathrm{b})$ : Localised contour representation of the dimensional $u$ velocity in the $\{X, z\}-$ plane for $L=10$ and $A=0.04$. Negative contours are highlighted for the region of flow separation about $X \in[0.425: 0.525]$. Arrows indicate the direction of the velocity vector field, while the solid black curve corresponds to the location that $99 \%$ of the $U$-velocity has been attained. (c): Pressure at the boundary layer edge associated with the chord region in (b), for the non-deformed (solid line) and wavy surface (dashed).

stream, the surface waviness induces a chordwise varying pressure gradient with favourable and adverse behaviour associated with the respective crests and troughs of the surface. For sufficiently large surface wave amplitudes, flow separation emerges within some of the troughs of the wavy surface. In figure $11(\mathrm{c})$ one relatively small section of reverse flow is observed about $X=0.475$, 


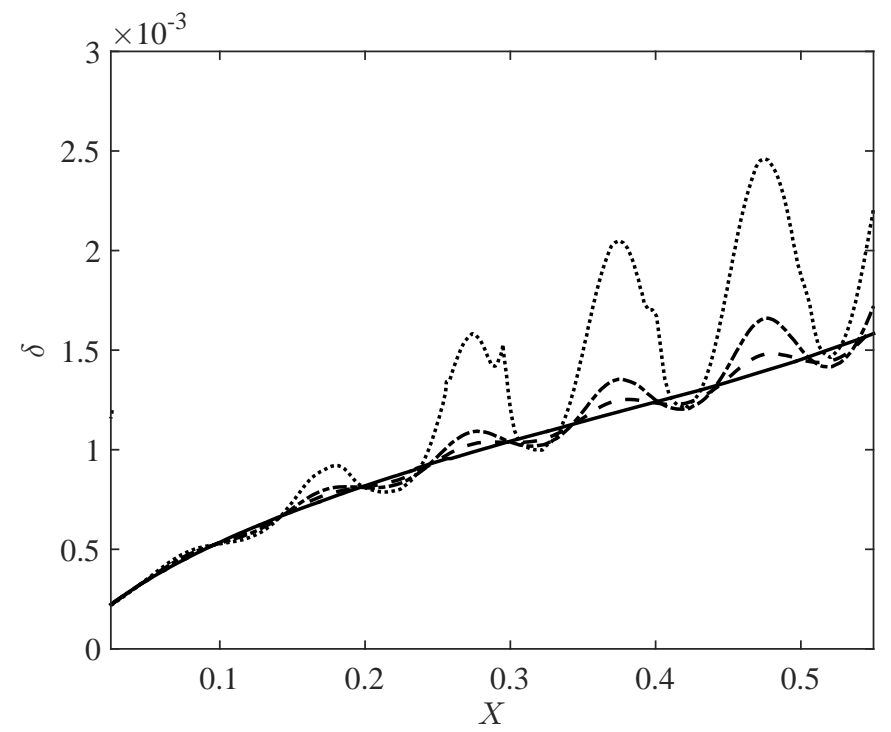

Fig. 13 Boundary layer thickness $\delta$ measured about $99 \%$ of the chordwise $U$-velocity for the four flows considered in figure 11.

while for the largest amplitude considered (figure 11(d)) three large regions of negative flow are established. In the latter illustration the flow initially separates about $X=0.25$, which is located along the downward curve of the sinusoidal wavy wall; the adverse pressure gradient has become sufficiently large to cause the flow to detach from the surface. The flow then reattaches to the wing about $X=0.3$ that is located along the upward slope of the wavy wall. As the flow passes downstream over further wavy sections, the region of separation grows substantially and the flow is almost entirely separated in the trough centred about $X \in[0.425: 0.525]$. Figure 12 highlights the flow structure about this location, with contour lines depicting the region of flow separation, while arrows indicate the direction and respective magnitudes of the velocity vector field. Flow separation is strongest near the upward curve of the wavy wall (contours about $\{X, z\}=\{0.49,0\}$ ), while the vector fields indicate that a fully formed region of flow circulation has developed with a centre of rotation about $\{X, z\}=\{0.48,0.0003\}$.

The solid black line in figure 12(a) represents the boundary layer thickness based on the wall distance that the $U$-velocity field has attained $99 \%$ of its maximum. At the peaks of the wavy wall, about $x=0.425$ and 0.525 , the boundary layer is approximately $1.5 \mathrm{~mm}$ thick. However, at the centre of the trough, $x=0.475$, the boundary layer is almost $2.5 \mathrm{~mm}$ thick. Thus, the formation of 
a separation bubble has caused a thickening of the boundary layer that has a maximum depth of about $1 \mathrm{~mm}$. The effect of surface waviness on the boundary layer thickness is further emphasised in figure 13. All four flow cases are included in the figure to illustrate the strong amplification of the boundary layer due to both the wavy wall and separation. About the surface troughs, the boundary layer thickness grows considerably, but about the surface peaks $\delta$ decreases to smaller amplitudes than that associated with the non-wavy wing (solid line). The figure also suggests that there is a relationship between the amplitude of the waviness and the magnification or reduction of the boundary layer. The $\delta$ variations were found to vary linearly with the amplitude $A$ about those locations where the boundary layer thickness decreases in size $(X=0.125,0.225$ etc. $)$ On the other hand, when $\delta$ increases $(X=0.175,0.275$ etc. $)$, the $\delta$ variations were found to be proportional to the square of $A$. However, this latter relationship is due to the flow separating within the troughs of the wavy surface for these particular flow systems (see figures $11(\mathrm{c}, \mathrm{d})$ ), causing $\delta$ to grow to larger magnitudes than what would be obtained if separation did not occur. This is confirmed by comparing boundary layer thicknesses for the flow systems with wavelengths $L=20$ and 40 , where amplitudes $A \leq 0.04$ were not large enough to cause separation. Though not shown here, the $\delta$ variations for these two surface wavelengths were always found to vary linearly with the amplitude $A$, independent of the chord location used to compare flow characteristics.

Figure 14 compares the $\mathrm{CF}$ velocity

$$
\frac{u_{C F}}{u_{e}}=U \sin \Lambda-V \cos \Lambda
$$

for the four surface variations considered in figures 11-13. The velocity profiles are drawn at four successive positions along the $X$-direction that are respectively centred at locations on the wavy surface with a positive gradient, crest, negative gradient and trough. On the crest of the surface wave (figure $14(\mathrm{~b})$ ), the $\mathrm{CF}$ velocity attains a larger magnitude as $A$ increases. Additionally, the maximum value of $u_{C F}$ has moved towards the wing wall. However, as the flow develops over the surface trench (figure $14(\mathrm{~d})$ ), large negative regions of $u_{C F}$ are observed for $A \geq 0.02$ that correspond to the regions of flow separation depicted in figure 11. Furthermore, the maximum positive magnitude of $u_{C F}$ decreases for larger $A$ and has now moved away from the surface.

The strength of the CF instability is measured using the CF Reynolds number $R_{C F}$, which is 

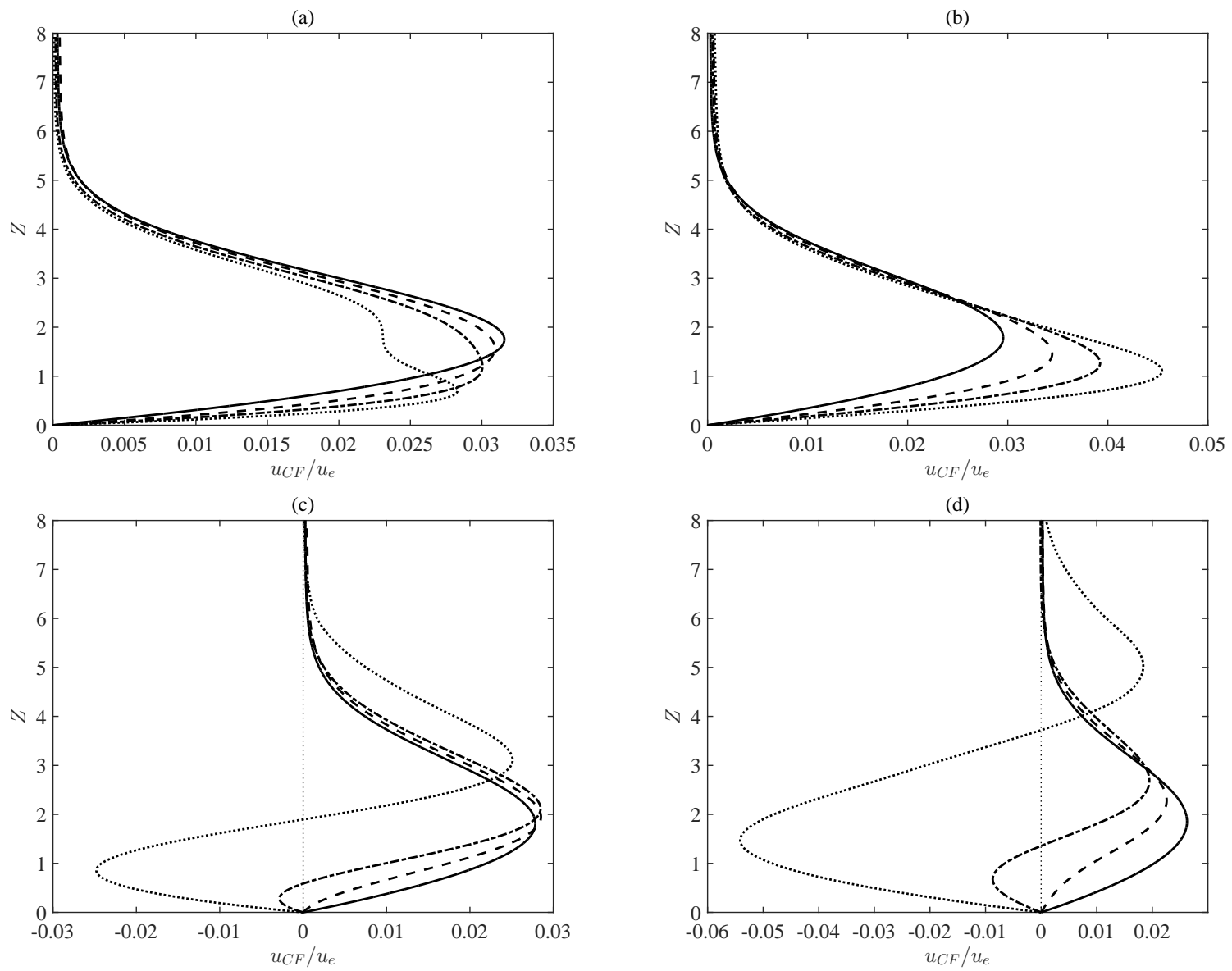

Fig. 14 Snapshots of the CF velocity $u_{C F}$ dimensionalised on $u_{e}$ for the four surface variations considered in figure 11, $A=0$, (solid line), $A=0.01$, (dashed), $A=0.02$, (chain) and $A=0.04$, (dotted). (a): $X=0.2$ (positive gradient); (b): $X=0.225$ (crest); (c): $X=0.25$ (negative gradient); (d): $X=0.275$ (trough).

calculated for those flow systems above (figures 11-14) and drawn in figure 15(a). For the surface geometry under consideration, $\{A, L, \lambda\}=\{A, 10,0\}$ and $A \leq 0.02, R_{C F}$ attains localised maximums about those locations corresponding to a peak of the surface wave $(X=0.125,0.225$ etc. $)$, while minimums are observed near the troughs of the wavy wing $(X=0.175,0.275$ etc. $)$ However, for the largest amplitude wave considered (dotted line), the size and variation of $R_{C F}$ is more complex, as the flow passes through large regions of separation. Thus, providing the local regions of separation are not too extreme, $\mathrm{CF}$ is enhanced by the positive slopes of the wave that precede the surface crests, where a stronger favourable pressure gradient is established.

The effect of surface waviness on the development of the CF disturbances was then considered 

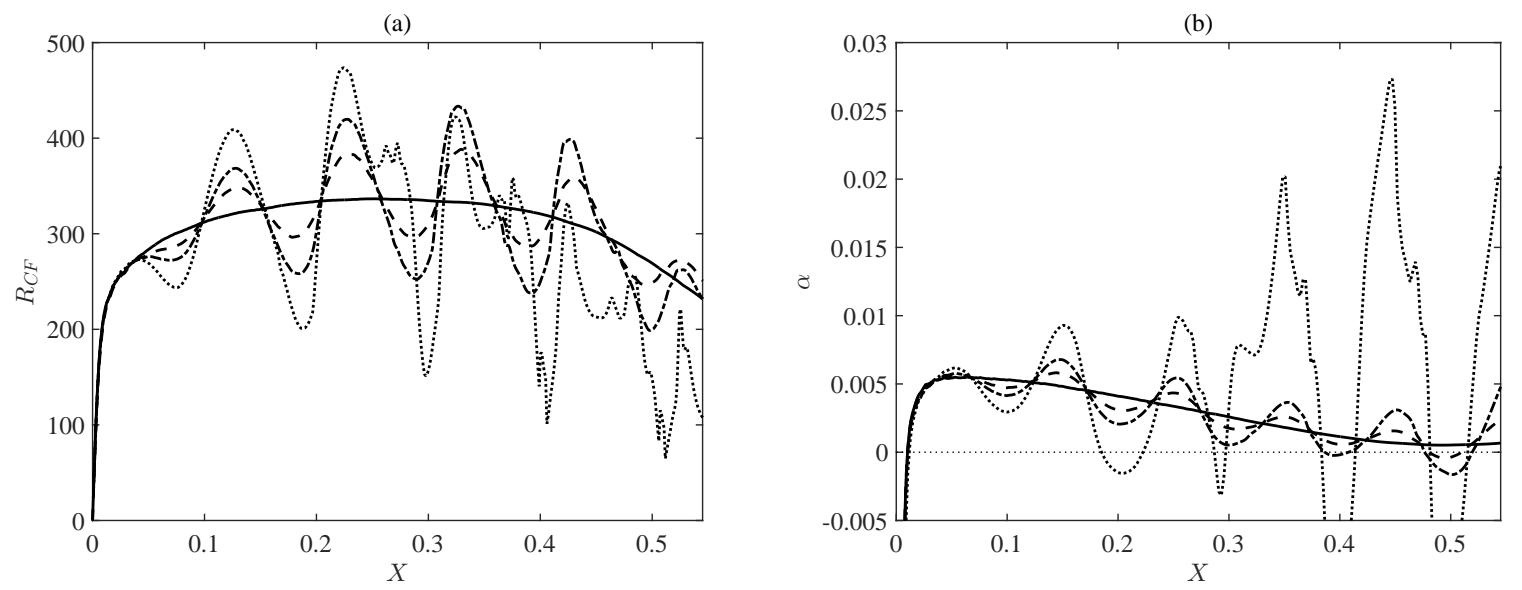

Fig. 15 (a): CF Reynolds number $R_{C F}$ and (b): Growth rate $\alpha$ of the CF disturbances generated for $\{\beta, f\}=\{2000,2000\}$, for the surface variations considered in figure 11, $A=0$, (solid line), $A=0.01$, (dashed), $A=0.02$, (chain) and $A=0.04$, (dotted).

by applying PSE methods to the boundary layers generated by REBL. The CF growth rates $\alpha$ were calculated for the parameter set $\{\beta, f\}=\{2000,2000\}$ and the solutions are illustrated in figure 15(b). The size of $\alpha$ fluctuates as the $\mathrm{CF}$ instability develops downstream, forming local maximums and minimums. However, in relation to $R_{C F}$, the peaks and dips in $\alpha$ are shifted by a quarter wavelength along the $X$-direction. Thus, the surface crest has enhanced the $\mathrm{CF}$ instability and established local maximum growth rates about the downward slopes of the wavy surface $(X=0.15,0.25$ etc.) The trough region of the wave then weakens the growth of the CF disturbance, forming a minimum $\alpha$ along the upwards slopes $(X=0.2,0.3$ etc. $)$

Figure 16 compares the $N$-factor calculations for four $\{\beta, f\}$ stability parameter sets. The strength of the CF instability is found to increase in plots 16(a) through to 16(d). For small amplitudes $A$ (dashed lines), the effect of waviness on the growth of the CF disturbance is relatively minor, with only marginal oscillatory variations in the $N$-factor. However, as the amplitude $A$ increases, the fluctuations in $N$ become relatively significant and the size of $N$ is found to grow considerably about the regions of flow separation. Additionally, the $N$-factor marginally decreases and increases about the surface crests and troughs, respectively. Thus, the growth in $R_{C F}$ about the crests of the surface enhances the amplification rate of the $\mathrm{CF}$ disturbance within the troughs of the wavy wall. 

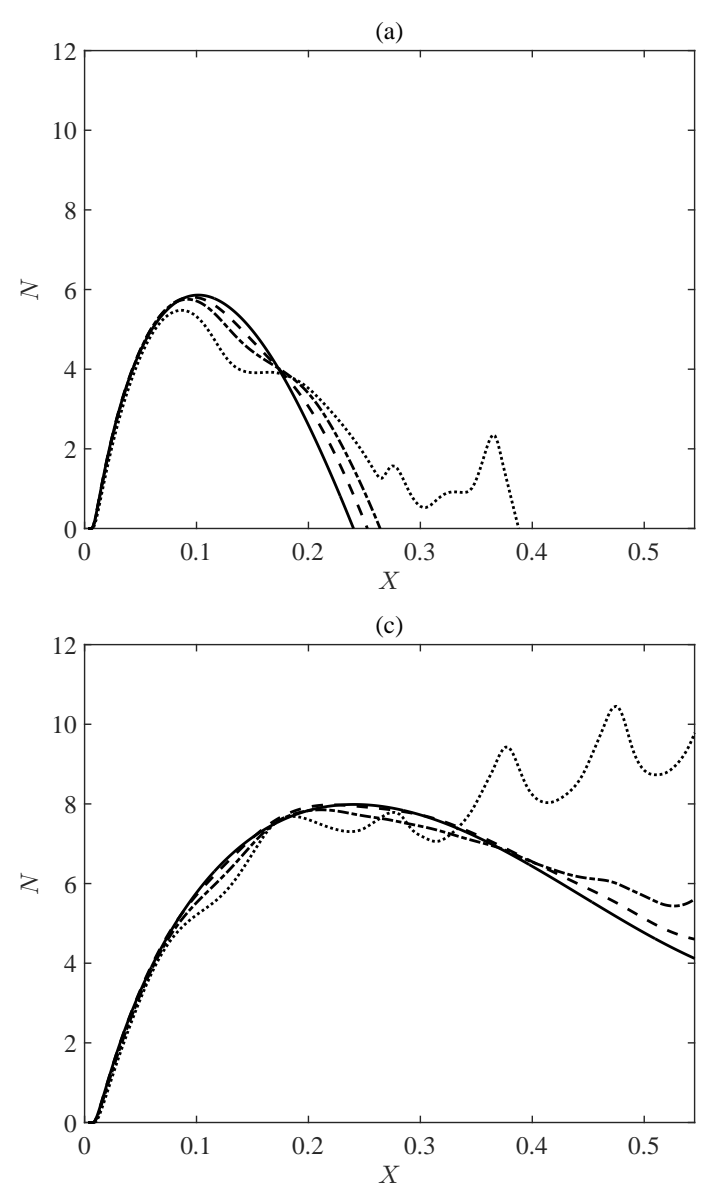
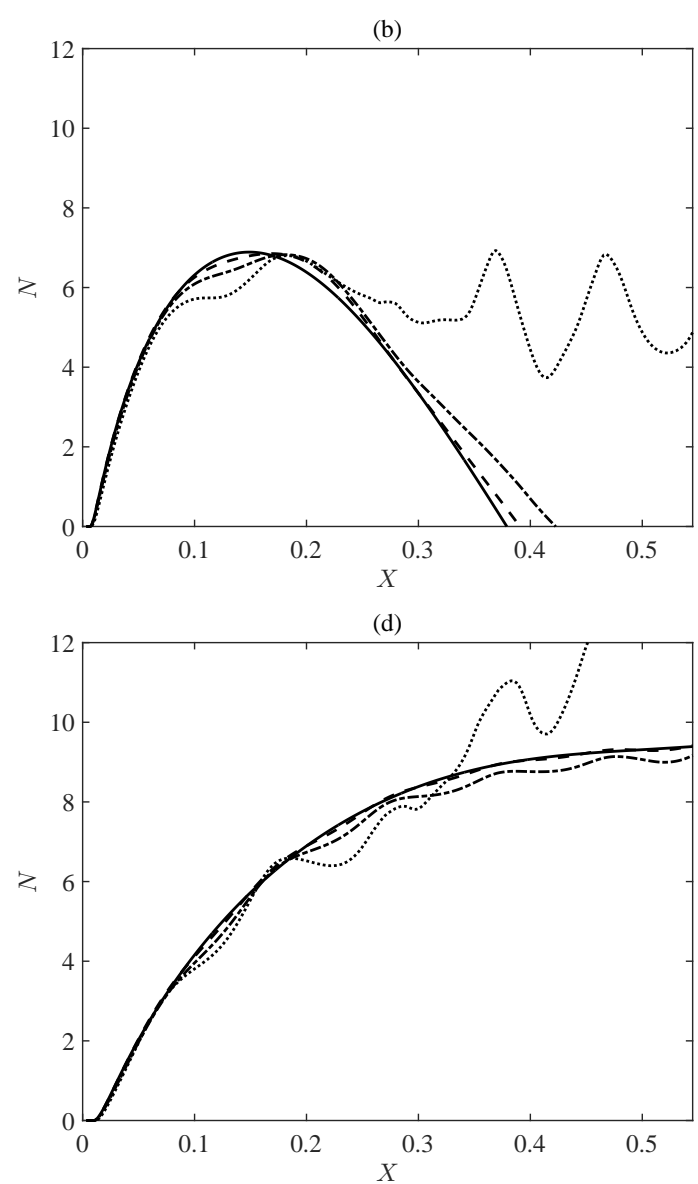

Fig. 16 PSE analysis for the flows with surface configurations described in figure 11, $A=0$, (solid line), $A=0.01,($ dashed), $A=0.02$, (chain) and $A=0.04$, (dotted). $N$-factor calculations for crossflow disturbances of wavelength $\beta\left(\mathbf{m}^{-1}\right)$ and frequency $f(\mathbf{H z}) ;(\mathbf{a}):\{\beta, f\}=\{5000,5000\}$; (b): $\{\beta, f\}=\{4000,4000\}$; (c): $\{\beta, f\}=\{3000,3000\}$; (d): $\{\beta, f\}=\{2000,2000\}$.

PSE analysis was then utilised to compute the CF amplification rates of several frequencies and wavenumbers. The resulting $N$-factors are plotted in figure 17 , where results based on CoBL solutions are drawn using dashed lines, while dotted lines with cross markers represent the equivalent calculations established using the boundary layers generated by the REBL extraction method. The agreement between the CoBL and REBL methods is again found to be excellent. The pressure gradient $P_{X}$ at the boundary layer edge is included within the illustration to further our understanding of the effect of surface waviness on the growth of the disturbances. For the surface configurations considered, a strong adverse pressure gradient develops along the downward slopes of the wavy wall (refer to the chord region $0.225 \leq X \leq 0.275$ ). As the amplitude $A$ increases, the pressure gradient 
(a)
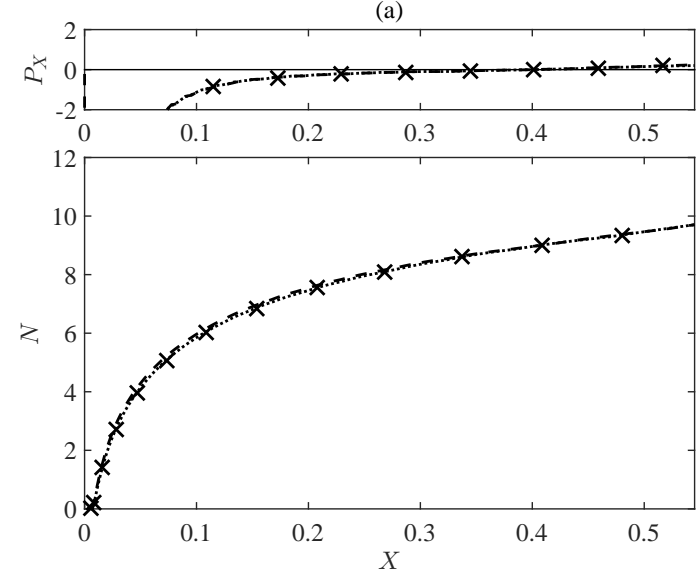

(c)
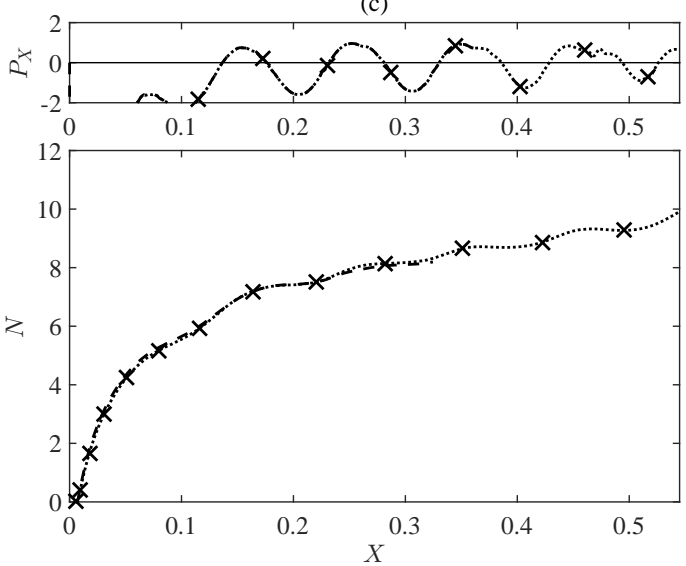

(b)
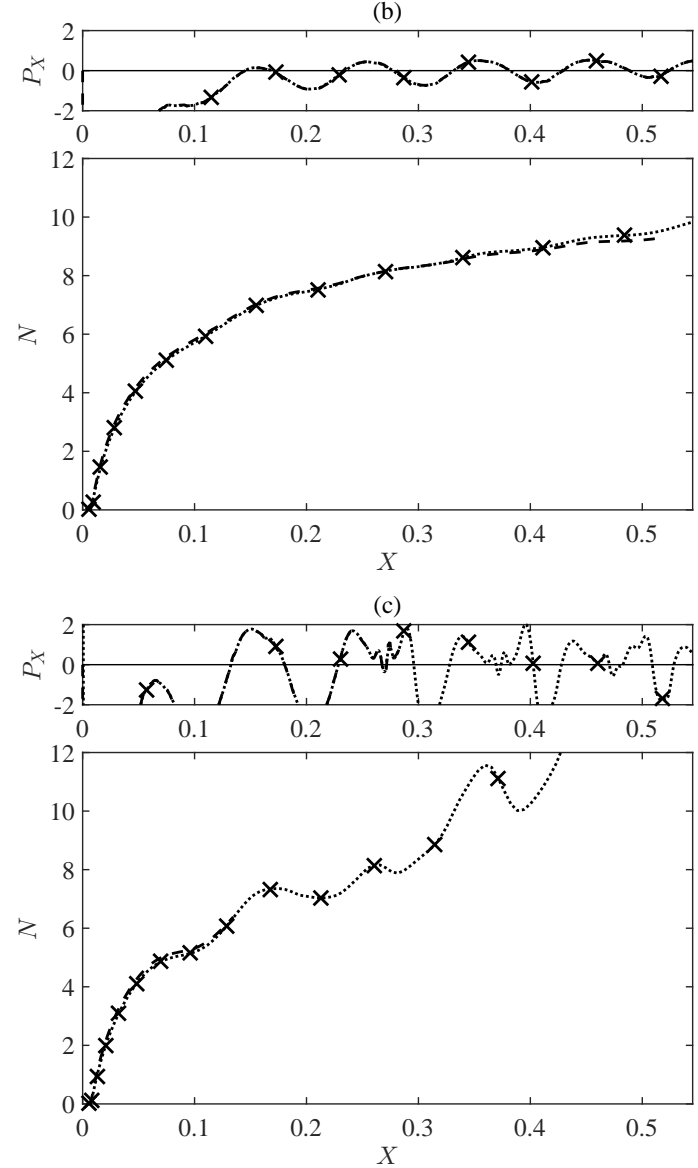

Fig. 17 PSE $N$-factor analysis corresponding to the wavy surface deformations described in figure 11. Dashed lines represent solutions based on CoBL calculations, while dotted lines with cross markers specify results given by REBL. The pressure gradients $P_{X}$ at the boundary layer edge are also included.

becomes increasingly complex and irregular, forming spike-like features about the locations where the boundary layer has separated.

For the first two illustrations in figure 17, the two sets of $N$-factor results are identical over the chord length shown. However, for the larger amplitude cases depicted in figure 17(c, d), the boundary layer method breaks down about the respective locations $X=0.3$ and 0.15 . Thus, the stability analysis of the CoBL generated boundary layer solutions is limited to the flow upstream of these chord positions. Nevertheless, using boundary layer solutions obtained from the new REBL scheme, stability analysis could be carried out for the full chord range under consideration. For the surface wave $A=0.02$ (figure $17(\mathrm{c})$ ), $N$-factor amplification rates were obtained for the entire chord 

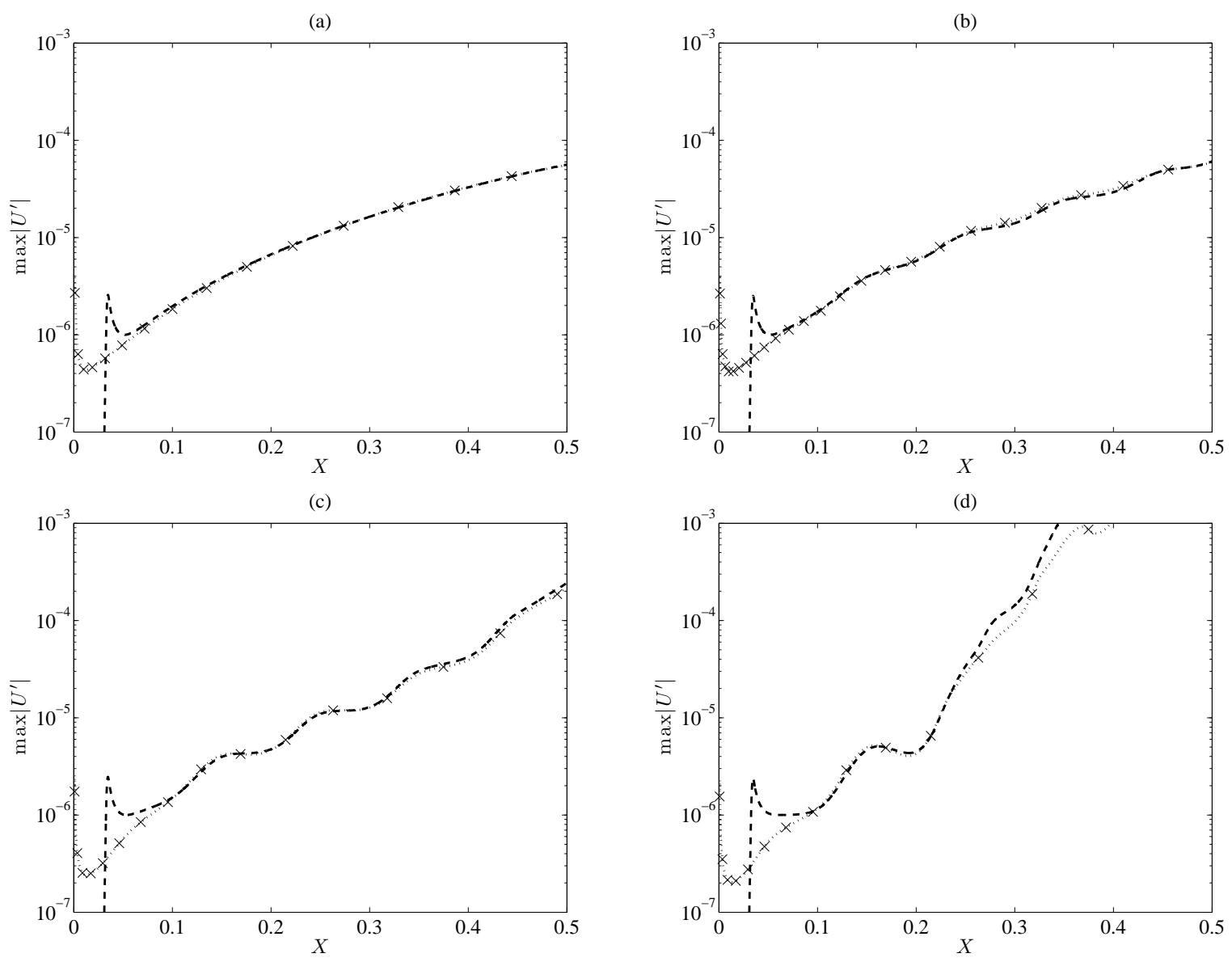

Fig. 18 Comparisons between PSE and LNS solutions of the $U^{\prime}$-velocity perturbation amplitude for a stationary crossflow disturbances of spanwise wavenumber $\beta=1000 \mathrm{~m}^{-1}$. The four flow systems correspond to those depicted in figure 11.

length shown, which includes the small pockets of flow separation that were documented in figure $11(\mathrm{c})$.

A complete stability analysis of the largest amplitude considered (figure 17(d)) was also obtained using the REBL generated boundary layer. This was achieved even though the pockets of flow separation are now very large. The growth of the CF instability is initially comparable in size and behaviour to the lower amplitude wavy wall systems. However, as the PSE analysis passes through the complex regions of flow separation, the development of the CF disturbance behaves very differently and grows to much larger $N$-factors than those seen previously. This would suggest that transition will appear upstream of that found for the other flow systems.

Figure 18 compares the maximum amplitudes of the $U^{\prime}$-velocity perturbation to the flow systems 
considered in figure 11. The illustrated results correspond to stationary CF disturbances with a spanwise wavenumber $\beta=1000 \mathrm{~m}^{-1}$. Dashed lines illustrate solutions given by the LNS scheme where a periodic wall forcing was centred about $X=0.03$. The sharp peak about this position illustrates the effective size of the forcing that excites the CF disturbance. Dotted lines with cross markers depict the equivalent results generated using PSE methods, which have been scaled to match the LNS solution at a point slightly downstream of the wall forcing. For the three cases shown in figures 18(a-c) the LNS and PSE results are almost identical, with comparable magnitudes and oscillatory behaviour (due to the wall waviness). Even for the largest amplitude considered (figure 18(d)), differences between the two stability methods only arise with the appearance of flow separation about $X=0.25$. Beyond this location the illustration suggests that the PSE approach marginally under predicts the relative sizes of the perturbation. The differences in the growth of the CF disturbance can be attributed to the large regions of separation that have formed for this particular flow system. As the PSE method is based on a streamwise marching procedure, the effect of separation along the upstream direction is ignored. Thus, for those wavy surface variations that generate complex separated flows, a stability analysis based on LNS or alternative methods may be more appropriate. Nevertheless, the PSE scheme is still very much applicable to those surface configurations considered in the subsequent sections where separation does not form or is not so large as to cause the stability variations observed in figure 18(d).

\section{Amplitude of Wave}

The effect of increasing the amplitude $A$ of the surface waviness is considered in figure 19, where the $N$-factor is again based on the strongest growing amplification rates of several disturbances. The difference $\Delta N$ between the $N$-factor results of the wavy wall configuration and that generated for the non-deformed surface are also illustrated. The phase from the leading edge is set to zero, four amplitudes $A$ are considered and the solutions for the wavelength $L=20$ are plotted in figure 19(a) and the wavelength $L=40$ are drawn in 19(b). For those cases considered here, waviness is found to have a stabilising effect over some surface regions and is marginally destabilising over other sections of the wing. For instance, $N$-factor solutions corresponding to the wavelength $L=20$ decrease about $0.15 \leq X \leq 0.25$, which coincides with a trough and peak respectively centred at 
(a)
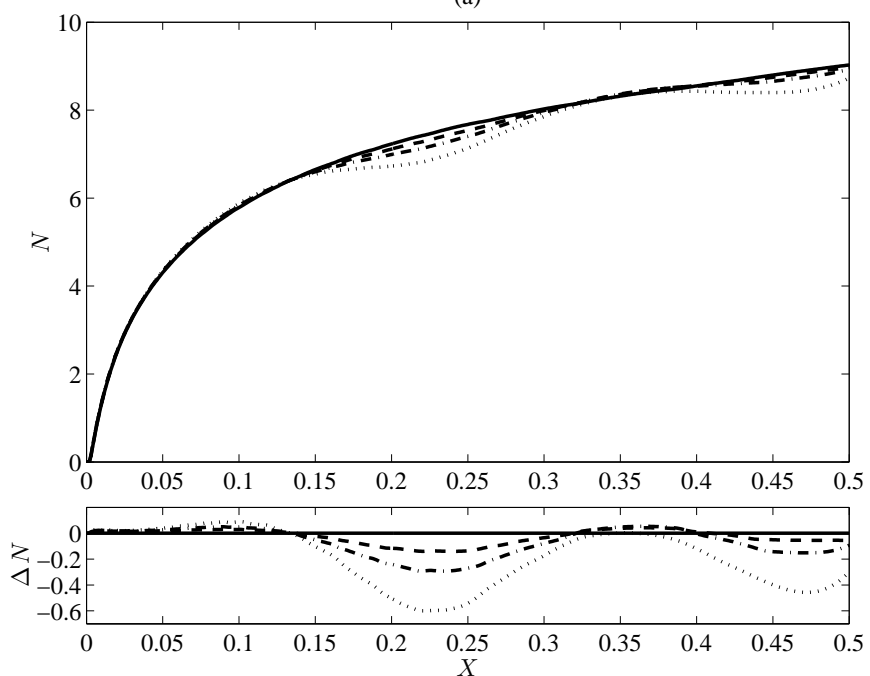

(b)
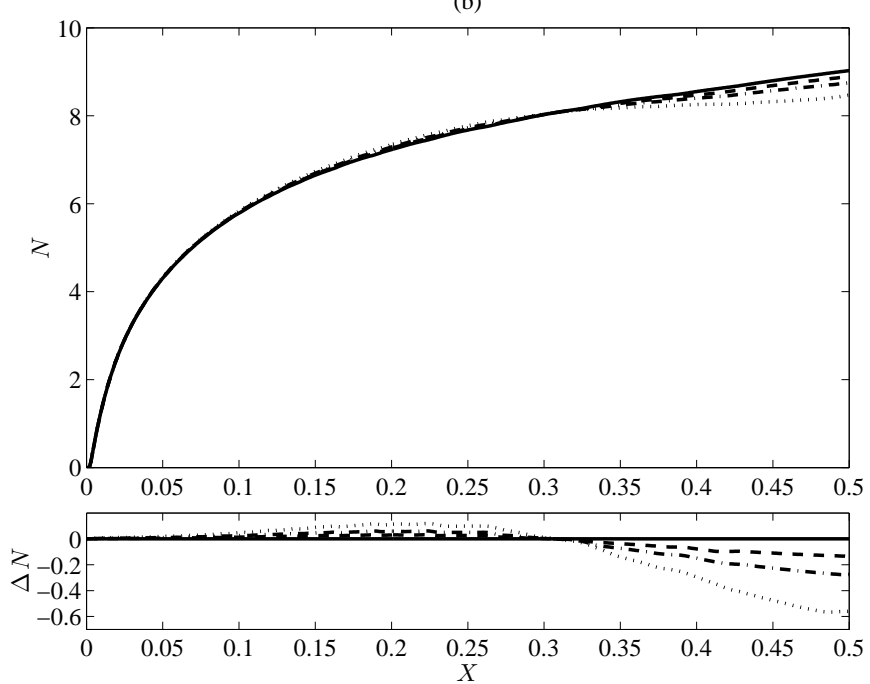

Fig. 19 Effect of the amplitude $A$ on the $N$-factor and differences $\Delta N$. (a): $L=20 ;$ (b): $L=40$ and $A=0.01$ (dashed), 0.02 (chain), 0.04 (dotted). Solid line represents the non-deformed solution.

$X=0.15$ and $X=0.25$. This particular observation is a direct consequence of the earlier analysis of $R_{C F}$, which is enhanced as the flow passes over the surface peaks, resulting in a larger growing $\mathrm{CF}$ instability about the troughs of the surface wave. Additionally, the variations $\Delta N$ are linearly proportional to the amplitude $A$ of the wavy surface. Thus, the linear variations in the boundary layer thickness $\delta$ that were observed for these particular wing configurations, are mirrored in the $N$ factor differences. This behaviour was observed for all cases considered, except for those flows where 
(a)

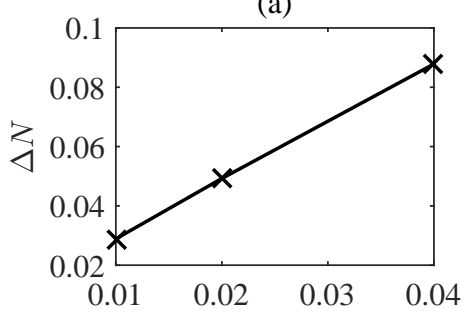

(c)

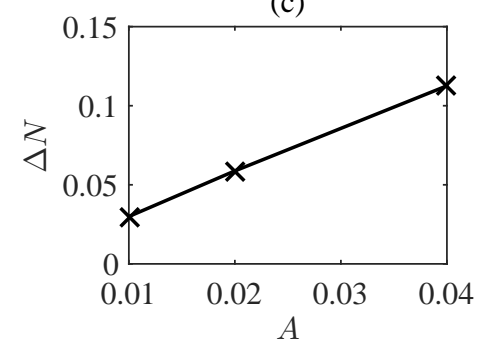

(b)

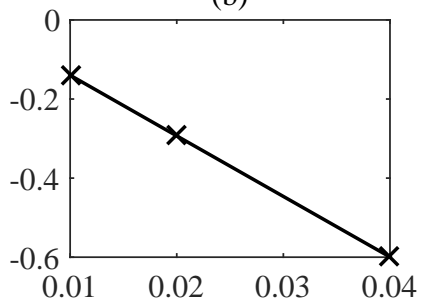

(d)

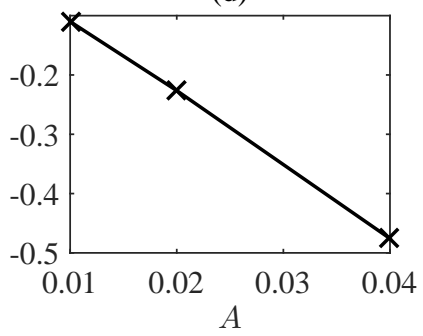

Fig. 20 Variation $\Delta N$ as a function of the amplitude $A$ for (a): $L=20$ and $X=0.1 ;(\mathbf{b}): L=20$ and $X=0.22 ;(\mathbf{c}): L=40$ and $X=0.2 ;(\mathbf{d}): L=40$ and $X=0.45$.

the boundary layer separated (refer to figures 11 through to 18). In these particular flow systems, separation caused significant amplification of the boundary layer, which resulted in stronger growing CF instabilities, where variations $\Delta N$ could no longer be related to $A$ using a simple linear model.

Figure 20 plots the variation $\Delta N$ as a function of the amplitude $A$, at various chord positions, for those flows considered in figure 19. The choice of parameters used to draw comparisons are outlined within the caption, where a cross marker indicates actual results. For all four illustrations, $\Delta N$ is found to vary linearly with $A$, where an increasing or decreasing variation is observed about the surface troughs or peaks, respectively.

\section{Length of Wave}

Figure 21 compares values of $N$ and $\Delta N$ for variable wavelengths $L$, for $A=0.02$ and $\lambda=0$. For those cases shown, varying the wavelength of the surface waviness is stabilising over large sections of the swept wing model. Furthermore, the illustration suggests that decreasing the wavelength has a stronger influence on the growth of the CF instability, whilst a reduced effect is observed for larger wavelengths. 


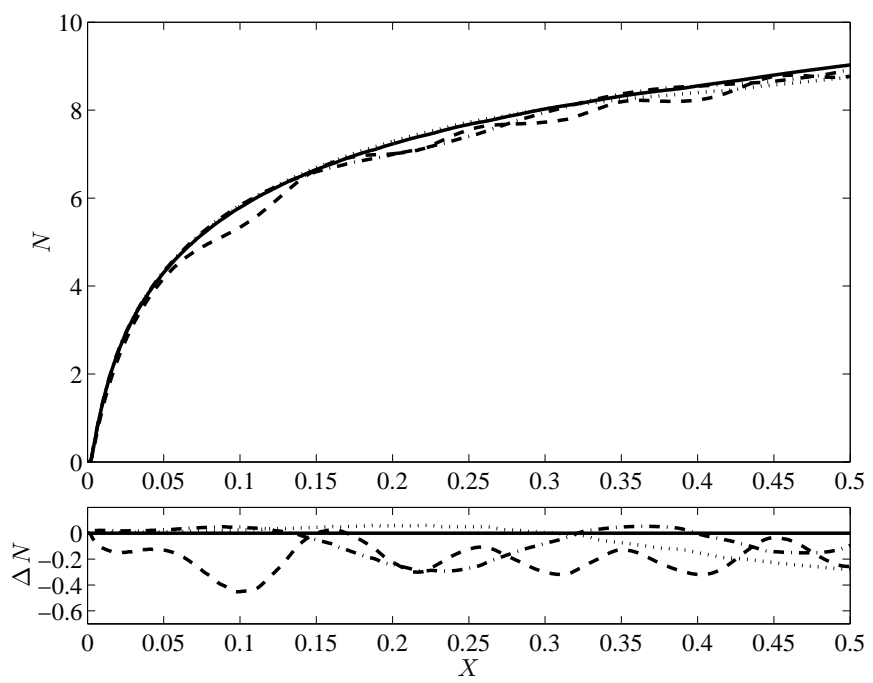

Fig. 21 Effect of the wave length on the $N$-factor and differences $\Delta N . A=0.02$ and $L=10$ (dashed), 20 (chain) ,40 (dotted). Solid line represents the non-deformed solution.

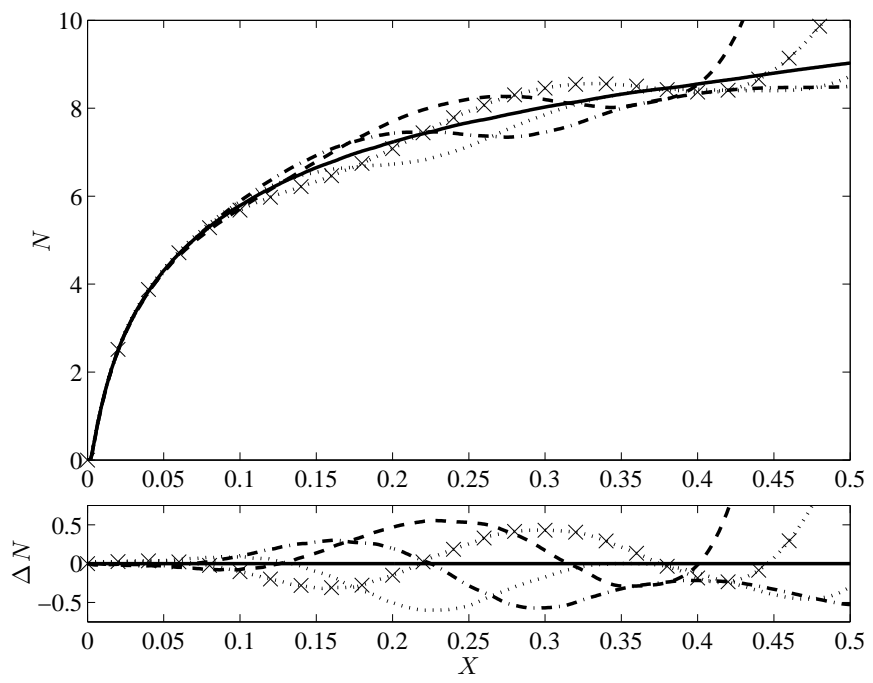

Fig. 22 Effect of phase on $N$-factor growth rate and differences $\Delta N . A=0.02$ and $L=20$. Phase $\lambda=-\pi / 2$ (chain), 0 (dotted), $\pi / 2$ (dotted with cross markers) and $\pi$ (dashed). Solid line represents the non-deformed solution.

\section{E. Phase of Wave}

Finally, the effect of varying the phase of the sinusoidal waviness on the CF instability is depicted in figure 22. Here the amplitude $A=0.02$ and the wavelength $L=20$ are fixed and the phase $\lambda$ 
(a)

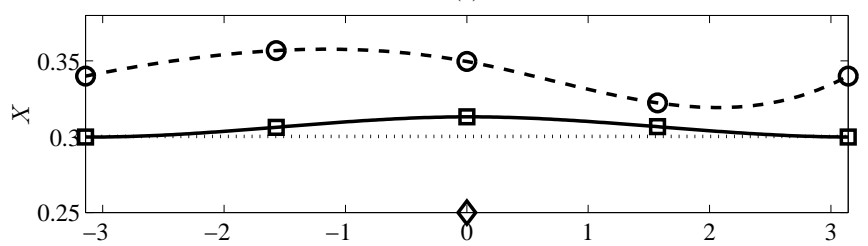

(b)

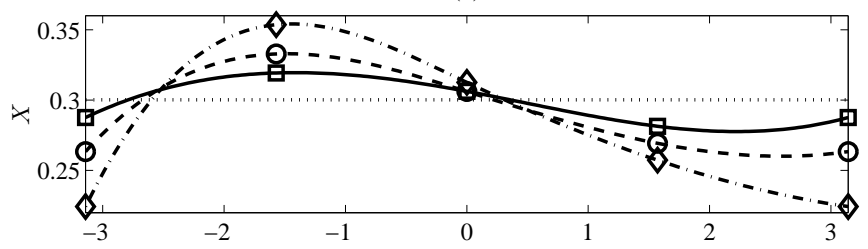

(c)

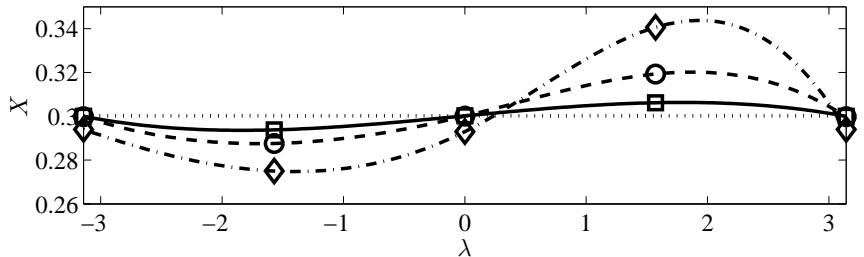

Fig. 23 Chord location $X$ that the CF instability attains a growth $N=8$ against phase shift $\lambda$. (a): $L=10$. (b): $L=20$. (c): $L=40$. Solid curves correspond to spline fitted data given for $A=0.01$, dashed $A=0.02$ and chain $A=0.04$. The various symbols denote actual stability calculations based on REBL boundary layers obtained from the RANS solutions. Dotted line illustrates the non-deformed solution.

from the leading edge is varied. The illustration suggests that the phase of the wavy surface has a significant impact on the growth of the disturbances. The case corresponding to $\lambda=0$ (dashed line) has the opposite effect of the case $\lambda=\pi$ (dotted line), with $N$ increasing in regions where the latter flow system suppresses the growth of the CF instability. This behaviour is due to the surface waviness (of the case $\lambda=0$ ) forming a trough where the second case $(\lambda=\pi)$ forms a crest. Similar behaviour is observed for the cases $\lambda= \pm \pi / 2$. Thus, the phase of the wavy wall has a significant role in the suppression or amplification rate of the $\mathrm{CF}$ disturbances.

\section{F. Summary of Wavy Surface Effects}

The effect of waviness on the growth of the CF disturbances is further illustrated in figure 23. Assuming that transition arises for $N=8$ (note that we only choose this value as a means 
of illustrating the influence of the surface waviness), the corresponding chord location is plotted as a function of $\lambda$ for variable $A$ and $L$. Symbols and spline fitting are used to draw results, while the dotted horizontal line in each subplot depicts the transition location corresponding to the nondeformed swept wing model. The illustration clearly shows that small amplitude wavy surfaces can cause relatively large differences in the growth of the $\mathrm{CF}$ disturbances, which may potentially give rise to significant variations in the onset of transition. Further, results indicate that the growth of the $\mathrm{CF}$ instabilities are significantly affected by the phase of the sinusoidal waviness. For the flow with wavelength $L=20$, phase shifts $\lambda \approx-\pi / 2$ are found to delay the onset of transition, while surfaces with $\lambda \approx \pi / 2$ promote transition. The opposite is true for wavelengths $L=40$, with the onset of $N=8$ found earlier for $\lambda \approx-\pi / 2$ and later for $\lambda \approx \pi / 2$. The change in the effect of the phase shift $\lambda$, for these two particular wavelengths, is due to a doubling of the wavelength $L$, which would suggest that the particular form of the surface waviness can be relatively beneficial along some sections of the swept wing, whilst having a negative impact about other regions of the surface.

\section{Conclusions}

A boundary layer method, REBL, for generating the laminar mean flow on an infinite swept wing model is described. Boundary layer characteristics, velocity profiles and the resulting $N$-factor computations of the CF disturbances based on PSE analysis are validated against solutions obtained from a compressible boundary layer scheme, with excellent agreement achieved for those cases that could be compared. REBL applies Paraview [31] filters and simple geometric transformations to extract the boundary layer directly from the solutions of the [26] solver. The undisturbed flow is then scaled and converted to a format suitable for stability analysis.

The effect of chordwise waviness of variable wavelength, amplitude and phase is considered and it is shown that the sinusoidally defined surface deformation can have a relatively large impact on the amplification rate of the $\mathrm{CF}$ disturbances. The $\mathrm{CF}$ Reynolds number $R_{C F}$ increases and decreases as the flow develops over the respective crests and troughs of the wavy surface. Localised minimums and maximums in the CF growth rate $\alpha$ are then located about positions corresponding to an upward and downward slope of the wavy wing, which establish negative and positive $N$-factor variations about the respective surface peaks and troughs. 
Smaller wavelengths are found to cause greater fluctuations of the $N$-factor, while variations in $N$ are observed to increase linearly with the amplitude $A$. This latter relationship is directly related to a similar linear relation between variations in the boundary layer thickness and $A$, for those flows where the boundary layer has not yet separated. Further, the phase of the waviness significantly affects the growth of the CF instabilities, as disturbances could either be suppressed or enhanced, depending on the configuration of the wavy wall. However, relationships between the variation $\Delta N$, the wavelength $L$ and the phase shift $\lambda$ were harder to formulate as the waviness was imposed over the full length of the wing model. As a possible future investigation, we may be able to formulate such expressions by implementing finite length wavy surfaces and comparing the growth of the CF instabilities at a downstream chord position. Hence, relationships comparable with the empirical correlation formula derived by Wie and Malik [17] may be formulated.

Although wavy walls cause variations in the $N$-factor amplification rate, the relative differences are quite small in comparison to previous observations of TS waves on a flat plate [17]. In that particular study, waviness was found to strongly destabilise disturbances and cause large changes in $N$. Although relatively large amplitude sinusoidal wave variations were considered here, (the largest being of size $0.4 \mathrm{~mm}$ compared with a boundary layer thickness of order $1-2 \mathrm{~mm}$ ), the effect on the CF development was relatively minor compared with the analysis on TS waves [17].

For sufficiently large amplitude to wavelength ratios (refer to figures 11 through to 19) the boundary layer was found to separate. Although conventional boundary layer methods breakdown when the flow separates, a thorough PSE and LNS analysis was still achieved by using flow solutions generated by the REBL extraction scheme. Furthermore, PSE analysis was successfully applied to separated flow systems, without the need for stepping over the pockets of separation. PSE results were also found to give very good agreement with the corresponding LNS computations, with both stability methods indicating that the CF disturbance grows substantially about the regions of separation. Thus, to avoid the large amplification caused by the pockets of separation, it may be necessary to ensure that the surface waviness is limited and restricted by a critical amplitude to wavelength ratio. 


\section{Acknowledgments}

We thank the referees for many helpful suggestions concerning the presentation and improvement of our results. This work has been supported by the EPSRC funded LFC-UK project: Development of Underpinning Technology for Laminar Flow Control, grant EP/I037946/1 and by the TSB funded Advanced Laminar Flow Enabling Technologies project.

\section{References}

[1] Ashworth, R. \& Mughal, S. M. "Modeling three dimensional effects on cross flow instability from leading edge dimples." Procedia IUTAM. Vol. 14, pp 201-210, 2015. IUTAM ABCM Symposium on Laminar Turbulent Transition. Ed. Medeiros, M. A. F. \& Meneghini, J. R. doi:10.1016/j.piutam.2015.03.041.

[2] Fage, A. "The smallest size of spanwise surface corrugation which affects boundary-layer transition on an airfoil," Aeronautical Research Council, $R$ \& M 2120, 1943.

[3] Carmichael, B. H., Whites, R. C. \& Pfenninger, W. "Low drag boundary layer suction experiments in flight on the wing glove of an F-94A airplane," Northrop Corp., Report No. NAI-57-1163 (BLC-101), 1957.

[4] Carmichael, B. H. "Surface waviness criteria for swept and unswept laminar suction wings," Northrop Corp., Report No. NOR-59-438 (BLC-123), 1959.

[5] Carmichael, B. H. \& Pfenninger, W. "Surface imperfection experiments on a swept laminar suction wing," Northrop Corp., Report No. NOR-59-454 (BLC-124), 1959.

[6] Holmes, B. J., Obara, C. J., Martin, G. L. \& Domack, C. S. "Manufacturing tolerances for natural laminar flow airframe surfaces," SAE Paper No. 850863, 1985.

[7] Obara, C. \& Holmes, B. J. "Flight-measured laminar boundary layer transition phenomena including stability theory analysis," NASA TP-2417, 1985.

[8] Wang, Y. X. \& Gaster, M. "Effect of surface steps on boundary layer transition," Exp. Fluids, Vol. 39, pp 679-686, 2005.

[9] Nayfeh, A. H., Ragab, S. A. \& Al-Maalitah, A. A. "Effect of bulges on the stability of boundary layers," Phys. Fluids, Vol. 31, No. 4, pp 796-806, 1988.

[10] Cebeci, T. \& Egan, D. A. "Prediction of transition due to isolated roughness," AIAA J., Vol. 27, 870-875, 1989.

[11] Masad, J. A. \& Iyer, V. "Transition prediction and control in subsonic flow over a hump," Phys. Fluids, Vol. 6, pp 313-327, 1994. 
[12] Masad, J. A. "Effect of surface waviness on transition in three-dimensional boundary-layer flow," $N A S A$ Contractor Report 201641, 1996.

[13] Smith, A. M. O. \& Gamberoni, N. "Transition, pressure gradient and stability theory," Douglas Airer. Co. Inc., ES 26388, 1956.

[14] Van Ingen, J. L. "A suggested semi-empirical method for the calculation of the boundary-layer transition region," Rep. Nos. VTH 71 and 74, Dept. Aeronaut. Eng., Univ. Technol., Delft, Neth. 1956.

[15] Mack, L. M. "Boundary-layer linear stability theory," AGARD Rep. No. 709, Von Kármán Inst., RhodeSt.-Genese, Belg. 1984.

[16] Poll, D. I. A. "Transition description and prediction in three-dimensional flows," AGARD Rep. No. 709, Von Kármán Inst., Rhode-St.-Genese, Belg. 1984.

[17] Wie, Y-S. \& Malik, M. R. "Effect of surface waviness on boundary-layer transition in two-dimensional flow," Comp. Fluids, Vol. 27, No. 2, pp 157-181, 1998.

[18] Herbert, T. "Parabolized stability equations," Annu. Rev. Fluid Mech., Vol. 29, 245-283, 1997.

[19] Gao, B., Park, D. \& Park, S. "Stability analysis of a boundary layer over a hump using parabolized stability equations," Fluid Dyn. Res. Vol. 43, p. 055503, 2011.

[20] Park, D. \& Park, S. O. "Linear and non-linear stability analysis of incompressible boundary layer over a two-dimensional hump," Comp. Fluids, Vol. 73, pp 80-96, 2013.

[21] Mughal, S. M. "Transition prediction in fully 3D compressible flows," Imperial College London Final report prepared for QinetiQ and British Aerospace (MAD), 2001.

[22] Kaups, K. \& Cebeci, T. "Compressible laminar boundary-layer with suction on swept and tapered wings," J. Aircraft, Vol. 14, No. 7, pp 661-667, 1977.

[23] Mughal, S. M. "Stability analysis of complex wing geometries: Parabolised stability equations in generalised non-orthogonal coordinates," AIAA Paper 2006-3222, 2006. doi: 10.2514/6.2006-3222

[24] Mughal, S. M. \& Ashworth, R. "Uncertainty quantification based receptivity modelling of crossflow instabilities induced by distributed surface roughness in swept wing boundary layers," AIAA Paper 2013-3106, 43rd AIAA Fluid Dynamics Conference, 2013. doi: 10.2514/6.2013-3106.

[25] Veldman, A. E. P. "New quasi-simultaneous method to calculate interacting boundary layers," $A I A A$ J., Vol. 19, pp 79-, 1981.

[26] "Technical documentation of the DLR TAU-code release 2011.2.0. Institute of Aerodynamics and Flow Technology, Braunshweig.

[27] Malik, M. R. "Hypersonic Flight Transition Data Analysis Using Parabolized Stability Equations with Chemistry Effects," J. Spacecraft Rockets, Vol. 40, No. 3, pp 332-344, 2003. 
[28] Liao, W., Malik, M. R., Lee-Rausch, E. M., Li, F., Nielsen, E. J., Buning, P. G., Choudhari, M. \& Chang, C. "Boundary-Layer Stability Analysis of the Mean Flows Obtained Using Unstructured Grids," J. Spacecraft Rockets, Vol. 52, No. 1, pp 49-63, 2015.

[29] Ashill, P. R., Beth, C. J. \& Gaudet, M. "A wind tunnel study of transitional flows on a swept panel wing at high subsonic speeds," CEAS 2nd European forum on laminar flow technology, 1996.

[30] Edwards, J. R. \& Chandra, S. "Comparison of Eddy-Viscosity Transport Turbulence Models for ThreeDimensional, Shock Separated Flowfields," AIAA J. Vol. 34, No. 4, 756-763, 1996.

[31] Ayachit, U. "The ParaView Guide: A Parallel Visualization Application", Kiteware, ISBN 9781930934306, 2015 . 